\title{
The mass ejection from the merger of binary neutron stars
}

\author{
Kenta Hotokezaka ${ }^{1}$, Kenta Kiuchi ${ }^{2}$, Koutarou Kyutoku ${ }^{3}$, Hirotada \\ Okawa $^{4}$, Yu-ichiro Sekiguchi ${ }^{2}$, Masaru Shibata ${ }^{2}$, and Keisuke Taniguchi ${ }^{5}$ \\ ${ }^{1}$ Department of Physics, Kyoto University, Kyoto 606-8502, Japan \\ ${ }^{2}$ Yukawa Institute of Theoretical Physics, Kyoto University, Kyoto 606-8502, Japan \\ ${ }^{3}$ Theory Center, Institute of Particles and Nuclear Studies, KEK, Tsukuba, Ibaraki 305-0801, Japan \\ ${ }^{4}$ CENTRA, Departamento de Física, Instituto Superior Técnico, \\ Universidade Técnica de Lisboa - UTL,Av. Rovisco Pais 1, 1049 Lisboa, Portugal \\ ${ }^{5}$ Graduate School of Arts and Sciences, The University of Tokyo, Tokyo 153-8902, Japan
}

\begin{abstract}
Numerical-relativity simulations for the merger of binary neutron stars are performed for a variety of equations of state (EOSs) and for a plausible range of the neutron-star mass, focusing primarily on the properties of the material ejected from the system. We find that a fraction of the material is ejected as a mildly relativistic and mildly anisotropic outflow with the typical and maximum velocities $\sim 0.15-0.25 c$ and $\sim 0.5-0.8 c$ (where $c$ is the speed of light), respectively, and that the total ejected rest mass is in a wide range $10^{-4}-10^{-2} M_{\odot}$, which depends strongly on the EOS, the total mass, and the mass ratio. The total kinetic energy ejected is also in a wide range between $10^{49}$ and $10^{51}$ ergs. The numerical results suggest that for a binary of canonical total mass $2.7 M_{\odot}$, the outflow could generate an electromagnetic signal observable by the planned telescopes through the production of heavy-element unstable nuclei via the $r$-process [1 3] or through the formation of blast waves during the interaction with the interstellar matter [4], if the EOS and mass of the binary are favorable ones.
\end{abstract}

PACS numbers: 04.25.Dm, 04.30.-w, 04.40.Dg

\section{INTRODUCTION}

Coalescence of binary neutron stars is one of the most promising sources for next-generation kilo-metersize gravitational-wave detectors such as advanced LIGO, advanced VIRGO, and KAGRA (LCGT) 5. These detectors will detect gravitational waves in the next 5 10 yrs. Statistical studies have predicted that the detection rate of gravitational waves emitted by binary neutron stars for these detectors will be $\sim 1-100$ per year [6, 7. The typical signal-to-noise ratio for most of these events will be $\sim 10$ or less. Thus, it will be quite helpful if electromagnetic or other signals observable are associated with the gravitational-wave bursts and the gravitational-wave detection is accompanied by the detections of other signals. Short-hard gamma-ray bursts (SGRB) have been inferred to be associated with the binary neutron star merger 8 . However, the jet of SGRB would be highly collimated [9, and hence, it will not be always possible to detect SGRB associated with the binary neutron star mergers. Moreover, it is not guaranteed that the telescopes for the observation of SGRB will be in operation with the gravitational-wave detectors. Exploring other possible signals that could be detected is a very important subject in the fields of gravitational-wave physics/astronomy [3, 4, 10, 13, 18.

This paper presents our latest results of numerical simulations performed in the framework of numerical relativity, focusing in particular on the exploration of the material ejected from binary neutron star mergers. In the past decade, numerical simulations for the merger of binary neutron stars in full general relativity, which is probably the unique approach of the rigorous theoretical study for this subject, have been extensively performed since the first success in 2000 [21] (see, e.g., [22, 23] for a review of this field). However, most of the simulations have focused on the studies of gravitational waveforms and the resulting product formed in the central region. Few attention has been paid to the study for the material ejected (but see 13 for a study in an approximate framework of general relativity, and see [14-17] for an early effort in the context of Newtonian gravity).

The material ejected from binary neutron star mergers may generate electromagnetic signals observable in the current and future-planned telescopes. One possible signal could be generated by the radioactive decay of unstable $r$-process nuclei, which are produced from the neutron-rich material in the ejecta [1, $\underline{3}, 12,13,17,18$. A fraction of the unstable nuclei produced subsequently decay in a short timescale and could heat up the ejecta, which emits a UV and visible light that may be observable by current and future-planned optical telescopes. In this case, the typical duration of a peak luminosity is expected to be several hours to a day. Another possible signal could be generated during the free expansion and the subsequent Sedov phase of the ejecta which sweeps up the interstellar medium and forms blast waves [4. In this process turning on, the shocked material at the blast waves could generate magnetic fields and accelerate particles that emit synchrotron radiation in the radio-wave band, for a hypothetical amplification of the electromagnetic field and a hypothetical electron injection. It is also pointed out that the binary neutron star merger could drive ultra-relativistic outflows in every direction and emit synchrotron radiation in x-ray-to-radio bands within a second-to-day timescale [19. All these stud- 
ies illustrate that exploring the process of the material ejection from binary neutron star mergers in detail is an important subject.

For the detailed numerical study of the ejected material, we have to be careful when following the motion of the materials in a low-density outer region. Most of the numerical-relativity simulations of binary neutron star mergers so far have been performed with a computational domain that was not wide enough for this purpose [22, 23]. We have to enlarge the computational domain sufficiently widely to confirm that the outflowed material is indeed ejected from the system (i.e., we have to confirm that the material is indeed unbound by the system by following the motion of the ejected material for a long time). Another subtle issue in the hydrodynamics simulations is that we have to put an artificial atmosphere when employing a conservative shock capturing scheme that is a standard one in this field 24]. In our previous simulations 25 27, we put an atmosphere with fairly large density $\left(\sim 10^{7} \mathrm{~g} / \mathrm{cm}^{3}\right)$ that did not affect the motion of neutron stars but did for the motion of the ejected material of low density which might escape to a far region. For the study of the mass ejection, we have to reduce the density of the atmosphere as low as possible (which should be much lower than the density of the ejected material), and in addition, we have to carefully confirm that such an artificial atmosphere does not affect the properties of the ejected material. In the simulation reported in this paper, we succeed in the simulation reducing the atmosphere density to a low level $\left(\lesssim 10^{5} \mathrm{~g} / \mathrm{cm}^{3}\right)$ enough to obtain a scientifically quantitative result.

The paper is organized as follows: In Sec. II, we summarize the equations of state (EOSs) employed and models of binary neutron stars. In Sec. III, we briefly summarize our formulation and numerics for solving Einstein's equation and hydrodynamics equations as well as the tools for diagnostics. In Sec. IV, numerical results are presented, focusing on the properties of the material ejected from the system. Section V is devoted to a summary and discussion. Throughout this paper, we employ the geometrical units $c=1=G$ where $c$ and $G$ are the speed of light and gravitational constant, respectively, although we recover $c$ when we need to clarify the units.

\section{EQUATIONS OF STATE AND CHOSEN MODELS}

In this section, we summarize the model EOSs employed in this paper, and initial condition of binary neutron stars chosen for numerical simulations. As shown in Sec. IV, the properties of the material ejected from binary neutron star mergers depend strongly on these inputs.

\section{A. Equations of state}

The exact EOS for the high-density nuclear matter is still unknown [28. This implies that a numerical simulation employing a single particular EOS, which might not be correct, would not yield a scientific result. A study, systematically employing a wide possible range of EOSs, is required for binary neutron star mergers. Nevertheless, the latest discovery of a high-mass neutron star PSR J1614-2230 with mass $1.97 \pm 0.04 M_{\odot}$ [29] significantly constrains the model EOS to be chosen, because it suggests that the maximum mass for spherical neutron stars for a given EOS has to be larger than $\sim 2 M_{\odot}$. This indicates that the EOS should be rather stiff, although there are still many candidate EOSs.

To model a variety of the candidate EOSs, specifically, we employ a piecewise polytropic EOS proposed by Read et al. 30. This EOS is described assuming that neutron stars are cold (in a zero-temperature state), i.e., the rest-mass density, $\rho$, determines all other thermodynamical quantities. To systematically model nuclear-theorybased EOSs at high density with a small number of parameters, the pressure is written in a parameterized form as

$$
P(\rho)=\kappa_{i} \rho^{\Gamma_{i}} \text { for } \rho_{i} \leq \rho<\rho_{i+1} \quad(0 \leq i \leq n),
$$

where $n$ is the number of the pieces used to parameterize a high-density EOS, $\rho_{i}$ is the rest-mass density at the boundary of two neighboring $(i-1)$-th and $i$-th pieces, $\kappa_{i}$ is the polytropic constant for the $i$-th piece, and $\Gamma_{i}$ is the adiabatic index for the $i$-th piece. Here, $\rho_{0}=0$, $\rho_{1}$ denotes a nuclear density $\sim 10^{14} \mathrm{~g} / \mathrm{cm}^{3}$ determined below, and $\rho_{n+1} \rightarrow \infty$. Other parameters $\left(\rho_{i}, \kappa_{i}, \Gamma_{i}\right)$ are determined by fitting with a nuclear-theory-based EOS. Requiring the continuity of the pressure at each $\rho_{i}, 2 n$ free parameters, say $\left(\kappa_{i}, \Gamma_{i}\right)$, determine the EOS completely. The specific internal energy, $\varepsilon$, and hence the specific enthalpy, $h$, are determined by the first law of thermodynamics and the continuity of each variable at boundary densities, $\rho_{i}$.

Read et al. 30] showed that a piecewise polytropic EOS with three pieces above the nuclear density (i.e., $n=3$ ) approximately reproduces most properties of the nucleartheory-based EOS at high density, and they derived the fitted parameters for a large number of nuclear-theorybased EOSs. In this paper, thus, we employ this piecewise polytropic EOS, determining the free parameters basically following [31 33. (in which a piecewise polytrope with $n=1$ was used). First, the EOS below the nuclear density $\rho_{1}$ is fixed by the following parameters

$$
\begin{aligned}
\Gamma_{0} & =1.35692395 \\
\kappa_{0} / c^{2} & =3.99873692 \times 10^{-8}\left(\mathrm{~g} / \mathrm{cm}^{3}\right)^{1-\Gamma_{0}} .
\end{aligned}
$$

The EOS for the nuclear matter was determined in 30 as follows: $\rho_{2}$ was fixed to be $\rho_{2}=10^{14.7} \mathrm{~g} / \mathrm{cm}^{3}$, and $P_{2}$ at $\rho=\rho_{2}$ was chosen as a free parameter. The reason 
TABLE I: Parameters and key quantities for four piecewise polytropic EOSs employed in this paper. $P_{2}$ is shown in units of dyn $/ \mathrm{cm}^{2} . M_{\max }$ is the maximum mass along the sequences of spherical neutron stars (cf. Fig. 2$) .\left(R_{1.35}, \rho_{1.35}\right)$ and $\left(R_{1.5}, \rho_{1.5}\right)$ are the circumferential radius and the central density of $1.35 M_{\odot}$ and $1.5 M_{\odot}$ neutron stars, respectively. We note that the values of the mass, radius, and density listed are slightly different from those obtained in the original tabulated EOSs (see the text for the reason). MS1 is referred to as this name in [30, but in other references (e.g., 28]), it is referred to as MS0. We follow 30] in this paper.

\begin{tabular}{ccccccc}
\hline EOS & $\left(\log \left(P_{2}\right), \Gamma_{1}, \Gamma_{2}, \Gamma_{3}\right)$ & $M_{\max }\left(M_{\odot}\right)$ & $R_{1.35}(\mathrm{~km})$ & $\rho_{1.35}\left(\mathrm{~g} / \mathrm{cm}^{3}\right)$ & $R_{1.5}(\mathrm{~km})$ & $\rho_{1.5}\left(\mathrm{~g} / \mathrm{cm}^{3}\right)$ \\
\hline APR4 & $(34.269,2.830,3.445,3.348)$ & 2.20 & 11.1 & $8.9 \times 10^{14}$ & 11.1 & $9.6 \times 10^{14}$ \\
ALF2 & $(34.616,4.070,2.411,1.890)$ & 1.99 & 12.4 & $6.4 \times 10^{14}$ & 12.4 & $7.2 \times 10^{14}$ \\
H4 & $(34.669,2.909,2.246,2.144)$ & 2.03 & 13.6 & $5.5 \times 10^{14}$ & 13.5 & $6.3 \times 10^{14}$ \\
MS1 & $(34.858,3.224,3.033,1.325)$ & 2.77 & 14.4 & $4.2 \times 10^{14}$ & 14.5 & $4.5 \times 10^{14}$ \\
\hline
\end{tabular}

is that $P_{2}$ is closely related to the radius and deformability of neutron stars 34. Namely, $P_{2}$ primarily determines the stiffness of an EOS. Second, $\rho_{3}$ was fixed to be $\rho_{3}=10^{15.0} \mathrm{~g} / \mathrm{cm}^{3}$. With these choices, the set of free parameters becomes $\left(P_{2}, \Gamma_{1}, \Gamma_{2}, \Gamma_{3}\right)$. These four parameters are determined by a fitting procedure (see [30] for the fitting procedure).

With the given values of $\Gamma_{1}$ and $P_{2}, \kappa_{1}$ and $\rho_{1}$ are subsequently determined by

$$
\begin{aligned}
\kappa_{1} & =P_{2} \rho_{2}^{-\Gamma_{1}}, \\
\rho_{1} & =\left(\kappa_{0} / \kappa_{1}\right)^{1 /\left(\Gamma_{1}-\Gamma_{0}\right)} .
\end{aligned}
$$

By the same method, $\kappa_{2}$ and $\kappa_{3}$ are determined from

$$
\kappa_{2} \rho_{2}^{\Gamma_{2}}=\kappa_{1} \rho_{2}^{\Gamma_{1}}, \quad \kappa_{3} \rho_{3}^{\Gamma_{3}}=\kappa_{2} \rho_{3}^{\Gamma_{2}} .
$$

Table 1 lists the EOSs and their parameters which we employ in this study. We choose four types of the representative EOSs. APR4 was derived by a variational method with modern nuclear potentials 35 for the hypothetical components composed of neutrons, protons, electrons, and muons; MS1 was derived by a mean-field theory for the hypothetical components composed of neutrons, protons, electrons, and muons, as well [36] ; H4 was derived by a relativistic mean-field theory including effects of hyperons 37; ALF2 is a hybrid EOS which describes a nuclear matter for a low density and a quark matter for a high density with the transition density is $3 \rho_{\text {nuc }}$ where $\rho_{\text {nuc }} \approx 2.8 \times 10^{14} \mathrm{~g} / \mathrm{cm}^{3}[38$. We note that the piecewise polytropic EOSs are slightly different from the original tabulated ones, because of their simple fitting formula. This results in a small error in the mass and radius of neutron stars. However, as shown in [30, the error is small (at most several percent), and the semiquantitative properties of the original EOSs are well captured by these simple EOSs.

Figure 1 plots the pressure as a function of the restmass density for four EOSs. APR4 has relatively small pressure for $\rho_{1} \leq \rho \lesssim \rho_{3}$ while it has high pressure for $\rho \gtrsim$ $\rho_{3}$. Thus, for $\rho<\rho_{3}$, which neutron stars of canonical mass $1.3-1.4 M_{\odot}$ have, this EOS is soft, and hence, the value of $P_{2}$ is relatively small. We note that for a small value of $P_{2}, \Gamma_{2}$ and/or $\Gamma_{3}$ have to be large $(\sim 3)$ because the maximum mass of spherical neutron stars, $M_{\max }$ for a given EOS has to be larger than $\sim 2 M_{\odot}$. Thus, an

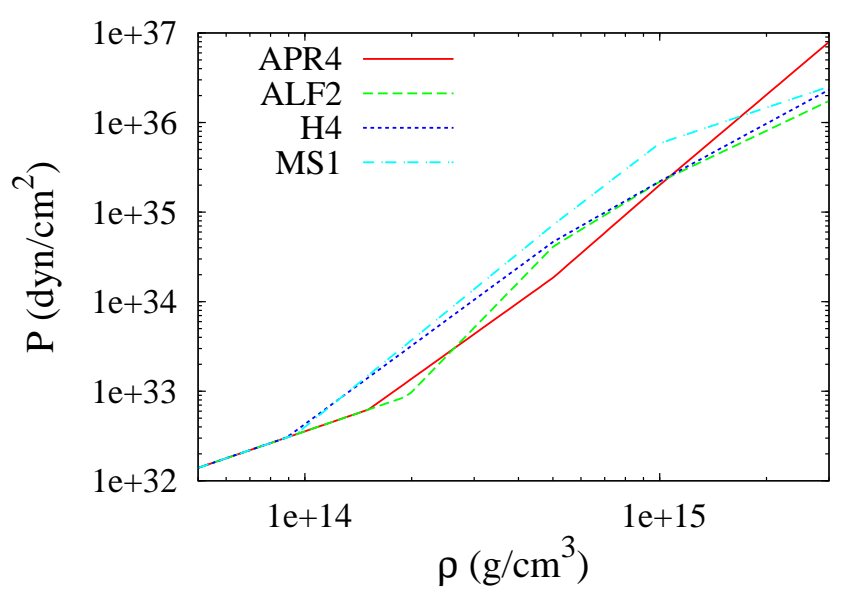

FIG. 1: Pressure as a function of the rest-mass density for APR4, ALF2, H4, and MS1 EOSs (the solid, dashed, dotted, and dash-dotted curves, respectively).

EOS that is soft at $\rho=\rho_{2}$ has to be in general stiff for $\rho \gtrsim \rho_{3}$. By contrast, $\mathrm{H} 4$ and MS1 have pressure higher than APR4 for $\rho \lesssim \rho_{3}$, although the EOSs become softer for a high-density region $\rho \gtrsim \rho_{3}$. In particular, MS1 has extremely high pressure (i.e., a higher value of $P_{2}$ ) among many other EOSs for $\rho \lesssim \rho_{3}$, and thus, it is the stiffest EOS as far as the canonical neutron stars are concerned. ALF2 has small pressure for $\rho \leq \rho_{2}$ as in the case of APR4, but for $\rho_{2} \lesssim \rho \leq \rho_{3}$, the pressure is higher than that for APR4. For $\rho \geq \rho_{2}$ the pressure of ALF2 is as high as that for $\mathrm{H} 4$. All the properties mentioned above are reflected in the radius, $R_{1.35}$, and central density, $\rho_{1.35}$, of spherical neutron stars with the canonical mass $M=1.35 M_{\odot}$ where $M$ is the gravitational (ArnowittDeser-Misner; ADM) mass of the spherical neutron stars in isolation: see Table If The pressure at $\rho=\rho_{2}\left(P_{2}\right)$ is correlated well with this radius and central density (see below).

Here, a word of caution is necessary for our APR4. The pressure in this piecewise polytropic EOS is extremely (unphysically) high in the high-density region with $\rho \gtrsim 10^{16} \mathrm{~g} / \mathrm{cm}^{3}$. This results pathologically in the situation that the sound velocity exceeds the speed of light for the high-density state. In reality, such a high density is achieved only in the formation of a black hole 
(i.e., inside the horizon), and such a pathology may not affect the evolution of the system for the outside of the horizon. However, this pathology could still break a numerical simulation after the formation of a black hole. To avoid this happens, we artificially set the maximum density as $10^{16} \mathrm{~g} / \mathrm{cm}^{3}$ when employing this EOS.

Figure 2 plots the gravitational mass as a function of the central density and as a function of the circumferential radius for spherical neutron stars for four EOSs. All the EOSs chosen are stiff enough that the maximum mass is larger than $1.97 M_{\odot}$. Because the pressure in a density region $\rho \lesssim 10^{15} \mathrm{~g} / \mathrm{cm}^{3}$ is relatively small (i.e., $P_{2}$ is small) for APR4 and ALF2, the radius for these EOSs is relatively small as $\sim 11 \mathrm{~km}$ and $12.5 \mathrm{~km}$, respectively, for the canonical mass of neutron stars $1.3-1.4 M_{\odot}$ [40]. By contrast, for $\mathrm{H} 4$ and MS1 for which $P_{2}$ is relatively large, the radius becomes a relatively large value 13.5 - $14.5 \mathrm{~km}$ for the canonical mass. The radius has also the correlation with the central density $\rho_{\mathrm{c}}$. For APR4 and ALF2 with $M=1.35 M_{\odot}$, $\rho_{\mathrm{c}} \approx 8.9 \times 10^{14} \mathrm{~g} / \mathrm{cm}^{3}$ and $\rho_{\mathrm{c}} \approx 6.4 \times 10^{14} \mathrm{~g} / \mathrm{cm}^{3}$. For H4 and MS1 with $M=1.35 M_{\odot}$, the central density is rather low as $\rho_{\mathrm{c}} \approx 5.5 \times 10^{14} \mathrm{~g} / \mathrm{cm}^{3}$ and $\rho_{\mathrm{c}} \approx 4.1 \times 10^{14} \mathrm{~g} / \mathrm{cm}^{3}$, respectively. As we show in Sec. IV] the properties of the material ejected from the merger of binary neutron stars depend strongly on the radius of the neutron stars or $\rho_{\mathrm{c}}$.

\section{B. Initial conditions}

We employ binary neutron stars in quasiequilibria for the initial condition of numerical simulations as in our series of papers [25, 26]. The quasiequilibrium state is computed in the framework described in 39 to which the reader may refer. The computation of quasiequilibrium states is performed using the spectral-method library LORENE 41.

Numerical simulations were performed, systematically choosing wide ranges of the total mass and mass ratio of binary neutron stars. Because the mass of each neutron star in the observed binary systems is in a narrow range between $\sim 1.2-1.45 M_{\odot}$ [40], we basically choose the neutron-star mass $1.20,1.25,1.30,1.35,1.40,1.45$, and $1.5 M_{\odot}$. Also, the mass ratio of the observed system $q:=$ $m_{1} / m_{2}(\leq 1)$ where $m_{1}$ and $m_{2}$ are lighter and heavier masses, respectively, is in a narrow range $\sim 0.85-1$. Thus, we choose $q$ as $0.8 \leq q \leq 1$. Specifically, the simulations were performed for the initial data listed in Table II

The initial data were prepared so that the binary has about $3-4$ quasicircular orbits before the onset of the merger. For four EOSs chosen, this requirement is approximately satisfied with the choice of the initial angular velocity $m \Omega_{0}=0.026$ for APR4 and ALF2 and $m \Omega_{0}=0.025$ for $\mathrm{H} 4$ and MS1. Here, $m=m_{1}+m_{2}$. For the following, the model is referred to as the name "EOS"-" $m_{1}$ " " $m_{2}$ "; e.g., the model employing APR4, $m_{1}=1.2 M_{\odot}$, and $m_{2}=1.5 M_{\odot}$ is referred to as model
APR4-120150.

\section{FORMULATION AND NUMERICAL METHODS}

Numerical simulations were performed using an adaptive-mesh refinement (AMR) code SACRA 42] (see also [43. for the reliability of SACRA). The formulation, the gauge conditions, and the numerical scheme are basically the same as those described in 42, except for the improvement in the treatment of the hydrodynamics code for a far region. Thus, we here only briefly review them and describe the present setup of the computational domain for the AMR algorithm and grid resolution.

\section{A. Formulation and numerical methods}

SACRA solves Einstein's evolution equations in the Baumgarte-Shapiro-Shibata-Nakamura formalism with a moving-puncture gauge [4]. It evolves a conformal factor $W:=\gamma^{-1 / 6}$, the conformal three-metric $\tilde{\gamma}_{i j}:=$ $\gamma^{-1 / 3} \gamma_{i j}$, the trace of the extrinsic curvature $K$, a conformally-weighted trace-free part of the extrinsic curvature $\tilde{A}_{i j}:=\gamma^{-1 / 3}\left(K_{i j}-K \gamma_{i j} / 3\right)$, and an auxiliary variable $\tilde{\Gamma}^{i}:=-\partial_{j} \tilde{\gamma}^{i j}$. Introducing an additional auxiliary variable $B^{i}$ and a parameter $\eta_{s}$, which we typically set to be $\approx 0.8 / m$ in units of $c=G=M_{\odot}=1$, we employ a moving-puncture gauge in the form [45]

$$
\begin{aligned}
\left(\partial_{t}-\beta^{j} \partial_{j}\right) \alpha & =-2 \alpha K \\
\left(\partial_{t}-\beta^{j} \partial_{j}\right) \beta^{i} & =(3 / 4) B^{i} \\
\left(\partial_{t}-\beta^{j} \partial_{j}\right) B^{i} & =\left(\partial_{t}-\beta^{j} \partial_{j}\right) \tilde{\Gamma}^{i}-\eta_{s} B^{i} .
\end{aligned}
$$

We evaluate the spatial derivative by a fourth-order central finite difference except for the advection terms, which are evaluated by a fourth-order lopsided upwind finite differencing scheme, and employ a fourth-order RungeKutta method for the time integration.

To solve hydrodynamics equations, we evolve $\rho_{*}:=$ $\rho \alpha u^{t} W^{-3}, \hat{u}_{i}:=h u_{i}$, and $e_{*}:=h \alpha u^{t}-P /\left(\rho \alpha u^{t}\right)$. Here, $u^{\mu}$ denotes the four velocity of the fluid. The advection terms are handled with a high-resolution central scheme by Kurganov and Tadmor [46] with a third-order piecewise parabolic interpolation for the cell reconstruction. We note that the total rest mass of the system is calculated by

$$
M_{*}=\int \rho_{*} d^{3} x .
$$

For the EOS employed in the numerical simulation, we decompose the pressure and specific internal energy into cold and thermal parts as

$$
P=P_{\text {cold }}+P_{\text {th }}, \varepsilon=\varepsilon_{\text {cold }}+\varepsilon_{\text {th }} .
$$



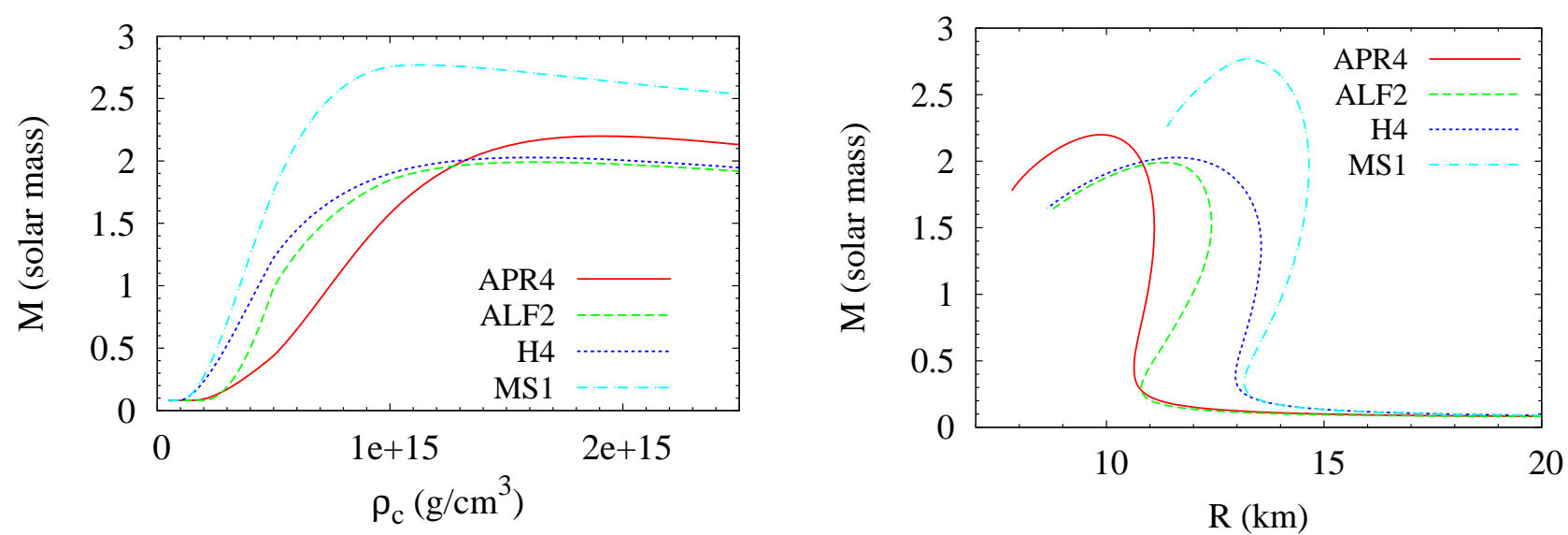

FIG. 2: Left: The gravitational mass as a function of the central density $\rho_{\mathrm{c}}$ for spherical neutron stars in APR4, ALF2, H4, and MS1 EOSs (the solid, dashed, dotted, and dash-dotted curves). Right: The same as the left panel but for the gravitational mass as a function of the circumferential radius.

We calculate the cold parts of both variables using the piecewise polytropic EOS (see section IIA) from the primitive variable $\rho$, and then the thermal part of the specific internal energy is defined from $\varepsilon$ as $\varepsilon_{\text {th }}=\varepsilon-\varepsilon_{\text {cold }}(\rho)$. Because $\varepsilon_{\text {th }}$ vanishes in the absence of shock heating, $\varepsilon_{\text {th }}$ is regarded as the finite temperature part determined by the shock heating in the present context. In this paper, we adopt a $\Gamma$-law ideal gas EOS for the thermal part as

$$
P_{\mathrm{th}}=\left(\Gamma_{\mathrm{th}}-1\right) \rho \varepsilon_{\mathrm{th}} .
$$

Following the conclusion of a detailed study in [47, $\Gamma_{\text {th }}$ is chosen in the range $1.6-2.0$ with the canonical value 1.8. For several models, we performed simulations varying the value of $\Gamma_{\mathrm{th}}$, and explored the effects of the shock heating; as shown in Sec. IV], numerical results depend fairly strongly on the value of $\Gamma_{\text {th }}$ (although the dependence on $\Gamma_{\text {th }}$ is not as strong as the dependence on the EOS, $\left.P_{\text {cold }}\right)$.

Because the vacuum is not allowed in any conservative hydrodynamics scheme (e.g., to derive the velocity by dividing the momentum density by the density), we put an artificial atmosphere of small density outside the neutron stars. The atmosphere has to be as tenuous as possible because a dense atmosphere may significantly affect the motion of the material ejected from binary neutron stars. Specifically, we set the density of the atmosphere in the following simple rule

$$
\rho_{\text {at }}= \begin{cases}f_{\text {at }} \rho_{\max } & \left(r \leq r_{\text {uni }}\right), \\ f_{\text {at }} \rho_{\max }\left(r / r_{\text {uni }}\right)^{-n} & \left(r \geq r_{\text {uni }}\right),\end{cases}
$$

where $\rho_{\max }$ denotes the maximum rest-mass density of the neutron stars at the initial state $\lesssim 10^{15} \mathrm{~g} / \mathrm{cm}^{3}$ (see Table II). We typically set $f_{\text {at }}=10^{-10}, n=3$, and $r_{\text {uni }}=16 L_{\min }$ where $2 L_{\min }$ denotes the side length of the finest computational domain in the AMR algorithm (see Sec. III C and Table III). For MS1, a computational region is wider and we set $f_{\text {at }}=10^{-11}$ to reduce the atmosphere mass. In these settings, the total rest mass of the atmosphere is always $\sim 10^{-6} M_{\odot}$ or less. In test simulations, we also adopted $n=2$ and $f_{\text {at }}=10^{-10}-10^{-12}$, and found that the numerical results on the ejected material such as its mass and its total energy depend only weakly on the values of $n$ and $f_{\text {at }}$ (e.g., the ejected mass increases by $\sim 10 \%$ if we change $n$ from 3 to 2 (denser one) for some models of APR4 and H4). Hence, we could safely conclude that the tenuous atmosphere chosen in this work does not significantly affect the properties of the ejected material.

We extracted $l=|m|=2$ modes of gravitational waves, $h_{+}$and $h_{\times}$, by calculating the outgoing part of the complex Weyl scalar $\Psi_{4}$ at finite coordinate radii $r \approx 200 M_{\odot}-400 M_{\odot}$ and by integrating $\Psi_{4}$ twice in time as in [33, to which the reader may refer (see also [48]). We also analyzed the evolution of gravitational-wave frequency, which is determined by extracting the phase of $\Psi_{4}, \arg \left(\Psi_{4}\right)$, and by taking the time derivative as $2 \pi f:=d\left(\arg \left(\Psi_{4}\right)\right) / d t$. To find the characteristic frequency of gravitational waves, we also define the average value of $f$ by

$$
f_{\text {ave }}:=\frac{\int f|h| d t}{\int|h| d t}
$$

where we used $|h|=\left(h_{+}^{2}+h_{\times}^{2}\right)^{1 / 2}$ as the weight factor. Then, we define the physical dispersion of $f$ by

$$
\sigma_{f}^{2}:=\frac{\int\left(f-f_{\text {ave }}\right)^{2}|h| d t}{\int|h| d t} .
$$

In the following, $f_{\text {ave }}$ and $\sigma_{f}$ are calculated for gravitational waves emitted by the remnant massive neutron stars. 
TABLE II: List of the parameters of the initial condition for binaries chosen in numerical simulations: Total mass, mass ratio, masses of two components, initial value of angular velocity, and initial frequency of gravitational waves $\left(f_{0}=\Omega_{0} / \pi\right)$.

\begin{tabular}{c|cccccc}
\hline Model & $m\left(M_{\odot}\right)$ & $q$ & $m_{1}\left(M_{\odot}\right)$ & $m_{2}\left(M_{\odot}\right)$ & $m \Omega_{0}$ & $f_{0}(\mathrm{~Hz})$ \\
\hline \hline APR4-130160 & 2.90 & 0.813 & 1.30 & 1.60 & 0.026 & 579 \\
APR4-140150 & 2.90 & 0.933 & 1.40 & 1.50 & 0.026 & 579 \\
APR4-145145 & 2.90 & 1.000 & 1.45 & 1.45 & 0.026 & 579 \\
APR4-130150 & 2.80 & 0.867 & 1.30 & 1.50 & 0.026 & 600 \\
APR4-140140 & 2.80 & 1.000 & 1.30 & 1.50 & 0.026 & 600 \\
APR4-120150 & 2.70 & 0.800 & 1.20 & 1.50 & 0.026 & 622 \\
APR4-125145 & 2.70 & 0.862 & 1.25 & 1.45 & 0.026 & 622 \\
APR4-130140 & 2.70 & 0.929 & 1.30 & 1.40 & 0.026 & 622 \\
APR4-135135 & 2.70 & 1.000 & 1.35 & 1.35 & 0.026 & 622 \\
APR4-120140 & 2.60 & 0.857 & 1.20 & 1.40 & 0.026 & 646 \\
APR4-125135 & 2.60 & 0.926 & 1.25 & 1.35 & 0.026 & 646 \\
APR4-130130 & 2.60 & 1.000 & 1.30 & 1.30 & 0.026 & 646 \\
\hline ALF2-140140 & 2.80 & 1.000 & 1.40 & 1.40 & 0.026 & 600 \\
ALF2-120150 & 2.70 & 0.800 & 1.20 & 1.50 & 0.026 & 622 \\
ALF2-125145 & 2.70 & 0.862 & 1.25 & 1.25 & 0.026 & 622 \\
ALF2-130140 & 2.70 & 0.929 & 1.30 & 1.40 & 0.026 & 622 \\
ALF2-135135 & 2.70 & 1.000 & 1.35 & 1.35 & 0.026 & 622 \\
ALF2-130130 & 2.60 & 1.000 & 1.30 & 1.30 & 0.026 & 646 \\
\hline H4-130150 & 2.80 & 0.867 & 1.30 & 1.50 & 0.025 & 577 \\
H4-140140 & 2.80 & 1.000 & 1.40 & 1.40 & 0.025 & 577 \\
H4-120150 & 2.70 & 0.800 & 1.20 & 1.50 & 0.025 & 598 \\
H4-125145 & 2.70 & 0.862 & 1.25 & 1.25 & 0.025 & 598 \\
H4-130140 & 2.70 & 0.929 & 1.30 & 1.40 & 0.025 & 598 \\
H4-135135 & 2.70 & 1.000 & 1.35 & 1.35 & 0.025 & 598 \\
H4-120140 & 2.60 & 1.000 & 1.30 & 1.30 & 0.025 & 621 \\
H4-125135 & 2.60 & 1.000 & 1.30 & 1.30 & 0.025 & 621 \\
H4-130130 & 2.60 & 1.000 & 1.30 & 1.30 & 0.025 & 621 \\
\hline MS1-140140 & 2.80 & 1.000 & 1.40 & 1.40 & 0.025 & 577 \\
MS1-120150 & 2.70 & 0.800 & 1.20 & 1.50 & 0.025 & 598 \\
MS1-125145 & 2.70 & 0.862 & 1.25 & 1.25 & 0.025 & 598 \\
MS1-130140 & 2.70 & 0.929 & 1.30 & 1.40 & 0.025 & 598 \\
MS1-135135 & 2.70 & 1.000 & 1.35 & 1.35 & 0.025 & 598 \\
MS1-130130 & 2.60 & 1.000 & 1.30 & 1.30 & 0.025 & 621 \\
\hline \hline
\end{tabular}

\section{B. Analysis of the ejected material}

In this section, we describe the method for analyzing the material ejected from the merger of binary neutron stars. Here, the ejected material is composed of a fluid element which is unbound by the gravitational potential of binary neutron stars and an object formed after the merger. Thus, first of all, we have to determine which fluid elements are unbound. To assess this point for all the fluid elements, we calculate $u_{\mu} t^{\mu}=u_{t}$ at each grid point. Here, $t^{\mu}$ is a timelike vector $(1,0,0,0)$ which is a Killing vector at spatial infinity. If $\left|u_{t}\right|>1$, we consider that the fluid element there is unbound.

Then we calculate the total rest mass, total energy (excluding gravitational potential energy), and total internal energy of the fluid element of $\left|u_{t}\right|>1$ by

$$
\begin{aligned}
M_{* \mathrm{esc}} & =\int_{\left|u_{t}\right|>1} \rho_{*} d^{3} x, \\
E_{\mathrm{tot}, \mathrm{esc}} & =\int_{\left|u_{t}\right|>1} T_{\mu \nu} n^{\mu} n^{\nu} \sqrt{\gamma} d^{3} x \\
& =\int_{\left|u_{t}\right|>1} \rho_{*} e_{*} d^{3} x, \\
U_{\mathrm{esc}} & =\int_{\left|u_{t}\right|>1} \rho_{*} \varepsilon d^{3} x,
\end{aligned}
$$

where $T_{\mu \nu}$ is the stress-energy tensor,

$$
T_{\mu \nu}=\rho h u_{\mu} u_{\nu}+P g_{\mu \nu},
$$

and $n^{\mu}$ is the unit timelike hypersurface normal. We note that the total energy is not uniquely defined by $E_{\text {tot,esc }}$ for dynamical spacetimes, and thus, the total energy defined here should be considered as an approximate measure for it. We here choose this expression for simplicity. We then define kinetic energy approximately by

$$
T_{* \text { esc }}:=E_{\text {tot,esc }}-M_{* \text { esc }}-U_{\text {esc }} .
$$


TABLE III: The grid structure for the simulation in our AMR algorithm. $\Delta x$ is the grid spacing in the finest-resolution domain with $L$ being the location of the outer boundaries along each axis and $L_{\text {min }}=N \Delta x . R_{\text {diam }} / \Delta x$ denotes the numbers of grid assigned inside the semi-major diameter of the lighter and heavier neutron stars in the finest level. $\lambda_{0}$ is the gravitational wavelength for the initial configuration. The last column shows the values of $\Gamma_{\text {th }}$ employed.

\begin{tabular}{c|cccccc}
\hline Model & $\Delta x(\mathrm{~km})$ & $R_{\text {diam }} / \Delta x$ & $L(\mathrm{~km})$ & $L_{\min }(\mathrm{km})$ & $\lambda_{0}(\mathrm{~km})$ & $\Gamma_{\text {th }}$ \\
\hline \hline APR4-130160 & 0.172 & 102,96 & 2636 & 10.3 & 518 & 1.8 \\
APR4-140150 & 0.167 & 102,101 & 2572 & 10.0 & 518 & 1.8 \\
APR4-145145 & 0.166 & 102,102 & 2550 & 10.0 & 518 & 1.8 \\
APR4-130150 & 0.172 & 102,98 & 2636 & 10.3 & 500 & 1.8 \\
APR4-140140 & 0.167 & 102,102 & 2572 & 10.0 & 500 & 1.8 \\
APR4-120150 & 0.172 & 103,98 & 2644 & 10.3 & 482 & $1.6,1.8,2.0$ \\
APR4-125145 & 0.174 & 102,100 & 2665 & 10.4 & 482 & 1.8 \\
APR4-130140 & 0.170 & 103,101 & 2609 & 10.2 & 482 & 1.8 \\
APR4-135135 & 0.169 & 102,102 & 2601 & 10.2 & 482 & $1.6,1.8,2.0$ \\
APR4-120140 & 0.174 & 102,99 & 2679 & 10.5 & 464 & 1.8 \\
APR4-125135 & 0.174 & 102,100 & 2665 & 10.4 & 464 & 1.8 \\
APR4-130130 & 0.171 & 102,102 & 2629 & 10.3 & 464 & 1.8 \\
\hline ALF2-140140 & 0.195 & 102,102 & 3001 & 11.7 & 500 & 1.8 \\
ALF2-120150 & 0.200 & 102,98 & 3065 & 12.0 & 482 & 1.8 \\
ALF2-125145 & 0.199 & 102,100 & 3054 & 11.9 & 482 & 1.8 \\
ALF2-130140 & 0.198 & 102,101 & 3044 & 11.9 & 482 & 1.8 \\
ALF2-135135 & 0.195 & 103,103 & 3001 & 11.7 & 482 & 1.8 \\
ALF2-130130 & 0.199 & 102,102 & 3054 & 11.9 & 464 & 1.8 \\
\hline H4-130150 & 0.222 & 102,98 & 3429 & 13.4 & 480 & 1.8 \\
H4-140140 & 0.219 & 102,102 & 3358 & 13.1 & 480 & 1.8 \\
H4-120150 & 0.228 & 102,96 & 3501 & 13.7 & 463 & $1.6,1.8,2.0$ \\
H4-125145 & 0.226 & 102,98 & 3465 & 13.5 & 463 & 1.8 \\
H4-130140 & 0.223 & 102,100 & 3430 & 13.4 & 463 & 1.8 \\
H4-135135 & 0221 & 102,102 & 3393 & 13.3 & 463 & $1.6,1.8,2.0$ \\
H4-120140 & 0.230 & 101,98 & 3537 & 13.8 & 446 & 1.8 \\
H4-125135 & 0.227 & 102,100 & 3494 & 13.6 & 446 & 1.8 \\
H4-130130 & 0.223 & 103,103 & 3430 & 13.4 & 446 & 1.8 \\
\hline MS1-140140 & 0.237 & 103,103 & 3644 & 14.2 & 480 & 1.8 \\
MS1-120150 & 0.249 & 101,97 & 3823 & 14.9 & 463 & 1.8 \\
MS1-125145 & 0.244 & 102,99 & 3751 & 14.7 & 463 & 1.8 \\
MS1-130140 & 0.244 & 101,100 & 3751 & 14.7 & 463 & 1.8 \\
MS1-135135 & 0.242 & 102,102 & 3715 & 14.5 & 463 & 1.8 \\
MS1-130130 & 0.244 & 102,102 & 3751 & 14.7 & 446 & 1.8 \\
\hline & & & & & &
\end{tabular}

We found irrespective of models that $T_{\text {*esc }}$ is much (by about $1-2$ orders of magnitude) larger than $U_{\text {esc }}$.

To approximately analyze the configuration of the ejected material, we also calculate the moments of inertia defined by

$$
I_{i i, \mathrm{esc}}=\int_{\left|u_{t}\right|>1} \rho_{*}\left(x^{i}\right)^{2} d^{3} x, \quad(\text { no sum for } i),
$$

and then, define

$$
\bar{X}=\sqrt{\frac{I_{x x, \mathrm{esc}}}{M_{* \mathrm{esc}}}}, \quad \bar{Y}=\sqrt{\frac{I_{y y, \mathrm{esc}}}{M_{* \mathrm{esc}}}}, \quad \bar{Z}=\sqrt{\frac{I_{z z, \mathrm{esc}}}{M_{* \mathrm{esc}}}},
$$

and $\bar{R}=\sqrt{\bar{X}^{2}+\bar{Y}^{2}}$. From $d \bar{R} / d t$ and $d \bar{Z} / d t$, we can determine the typical (average) velocity of the ejected material, which is denoted by $\bar{V}_{\text {esc }}^{R}$ and $\bar{V}_{\text {esc }}^{Z}$ in the following.

We consider a model that the configuration of the ejected material is approximated by an axisymmetric anisotropic shell of uniform density as

$$
\rho= \begin{cases}\rho_{\mathrm{esc}} & \pi / 2-\theta_{0} \leq \theta \leq \pi / 2+\theta_{0} \\ & \text { and } R_{-} \leq r \leq R_{+}, \\ 0 & \text { otherwise }\end{cases}
$$

where $\rho_{\mathrm{esc}}, R_{ \pm}$, and $\theta_{0}$ are time-varying parameters. In this case,

$$
\begin{aligned}
M_{* \mathrm{esc}} & =\frac{4 \pi}{3} \rho_{\mathrm{esc}}\left(R_{+}^{3}-R_{-}^{3}\right) \sin \theta_{0}, \\
\bar{R}^{2} & =\frac{1}{5} \frac{R_{+}^{5}-R_{-}^{5}}{R_{+}^{3}-R_{-}^{3}}\left(3-\sin ^{2} \theta_{0}\right), \\
\bar{Z}^{2} & =\frac{1}{5} \frac{R_{+}^{5}-R_{-}^{5}}{R_{+}^{3}-R_{-}^{3}} \sin ^{2} \theta_{0} .
\end{aligned}
$$

Thus for an axial ratio,

$$
\eta_{R}=\frac{\bar{Z}}{\bar{R}},
$$


$\sin \theta_{0}$ is calculated as

$$
\sin ^{2} \theta_{0}=\frac{3 \eta_{R}^{2}}{1+\eta_{R}^{2}}
$$

Hence, from the axial ratio calculated for a numerical result of the ejected material, we can approximately define the extent in the $\theta$ direction; e.g., for $\eta_{R}=0.4$ and 0.5 , $\theta_{0} \approx 40^{\circ}$ and $51^{\circ}$, respectively.

\section{Setup of AMR grids}

An AMR algorithm implemented in SACRA can prepare a fine-resolution domain in the vicinity of compact objects as well as a sufficiently wide domain that covers a local wave zone. In the present study, we prepare additional domains wider than those used in our previous studies [26, 32, 33], to follow the motion of the material ejected during the merger of binary neutron stars for a sufficiently long time (longer than $10 \mathrm{~ms}$ ).

The chosen AMR grids consist of a number of computational domains, each of which has the uniform, vertexcentered Cartesian grids with $(2 N+1,2 N+1, N+1)$ grid points for $(x, y, z)$ with the equatorial plane symmetry at $z=0$. Since we chose that the grid spacing for three directions is identical, the shape of each AMR domain is a half cube. We chose $N=60$ for the best resolved runs in this work, and all the results shown in the following were obtained with this resolution. We also performed simulations with $N=40$ and 50 (or 48) for several chosen models to check the convergence of the results (see Appendix A).

We classify the domains of the AMR algorithm into two categories: one is a coarser domain, which covers a wide region including both neutron stars with its origin fixed at the approximate center of mass throughout the simulation. The other is a finer domain, two sets of which comove with two neutron stars and cover the region in their vicinity. We denote the side length of the largest domain, number of the coarser domains, and number of the finer domains by $2 L, l_{c}$, and $2 l_{f}$, respectively. In this work, $l_{c}=5$ and $l_{f}=4$ (in total, 13). The grid spacing for each domain is $h_{l}=L /\left(2^{l} N\right)$, where $l=0-$ $l_{\max }\left(=l_{c}+l_{f}-1\right)$ is the depth of each domain. In the following, we denote $L / 2^{l_{\max }}$ by $L_{\min }$ and $h_{l_{\max }}$ by $\Delta x$.

Table III summarizes the parameters of the grid structure for the simulations. For all the simulations, $L$ is set to be $L / c \gtrsim 10 \mathrm{~ms}$. This implies that the material cannot escape from the computational domain in $\sim 10 \mathrm{~ms}$ after the onset of the merger, even if it could move with the speed of light. In reality, the speed of most of the ejected material is smaller than $\sim 0.5 c$, and thus, the material stays in the second coarsest level for more than $10 \mathrm{~ms}$. $L$ is also much larger than the gravitational wavelengths at the initial instant $\lambda_{0}:=\pi / \Omega_{0}$. This implies that a spurious effect caused by outer boundaries when extracting gravitational waves is excluded in the present work more efficiently than in the previous works. The semi-major diameter of each neutron star is covered approximately by 100 grid points for $N=60$.

\section{NUMERICAL RESULTS}

Table IV summarizes the remnant formed, the rest mass and kinetic energy of the ejected material measured at $10 \mathrm{~ms}$ after the onset of the merger $t=t_{\text {merge }}$, and the characteristic (average) frequency of gravitational waves emitted by the hypermassive neutron star (HMNS) for $N=6061$. Here, $t_{\text {merge }}$ is chosen to be the time at which the amount of the rest mass of the ejected material steeply increases. In the following two subsections, we summarize the results for the formation of HMNSs and black holes separately.

\section{A. Properties of the merger and mass ejection: HMNS case}

Binary neutron stars in quasicircular orbits evolve due to the gravitational-wave emission. Their orbital separation decreases gradually, and eventually, the merger sets in. Previous studies (e.g., 26]) clarified that soon after the onset of the merger, either a long-lived HMNS or a black hole is formed. For most of the simulations in this paper performed with stiff EOSs and with the canonical total mass $2.6-2.8 M_{\odot}$, we found that a long-lived HMNS is formed with its lifetime much longer than its dynamical timescale $\sim 0.1 \mathrm{~ms}$ and its rotation period $\sim 1 \mathrm{~ms}$; the lifetime is longer than $10 \mathrm{~ms}$ for most of the models employed in this paper. In this section, we pay attention to the case that such a HMNS is formed.

Figures 3-5 display snapshots of the density profiles in the merger for models APR4-135135, APR4-120150, and H4-120150, respectively. Figure 6 also displays the central density as a function of time for the models with $m_{1}=m_{2}=1.35 M_{\odot}$ (left), and $m_{1}=1.2 M_{\odot}$ and $m_{2}=1.5 M_{\odot}$ (right). These figures show that a compact and nonaxisymmetric object (proto HMNS) is formed in the central region soon after the onset of the merger. The shape and compactness of the HMNS depend strongly on the EOS and mass ratio; e.g., the presence of the asymmetric spiral arms found in the top panels of Figs. 4 and 5 is the feature only for the asymmetric binaries; the amplitude of the quasiradial oscillation is larger for the equalmass binaries; a high-amplitude quasiradial oscillation is a unique property found only for models with APR4 (see Fig. 6). However, it is universal that the HMNSs are rapidly rotating and nonaxisymmetric, irrespective of the EOS, total mass $\left(m \leq 2.8 M_{\odot}\right)$, and mass ratio, as found in previous studies [21, 25, 26]. This rapid rotation together with the nonaxisymmetric configuration not only results in the emission of strong gravitational waves but also is the key for an efficient mechanism of angular momentum transport from the HMNS to the surrounding material because the HMNS exerts the torque. 


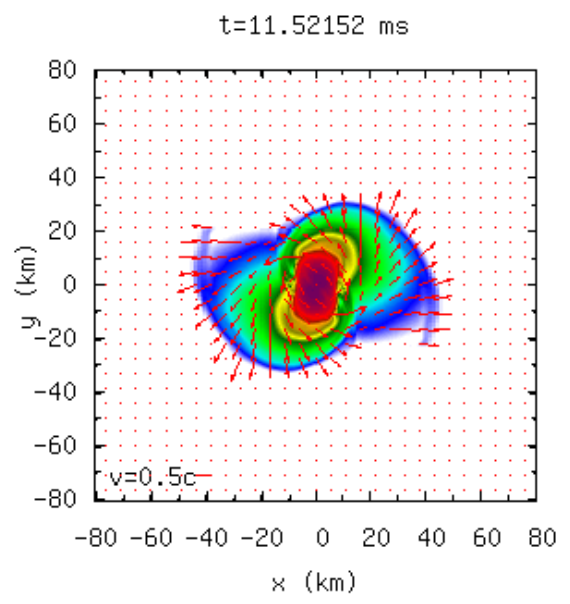

$\mathrm{t}=13.55473 \mathrm{~ms}$
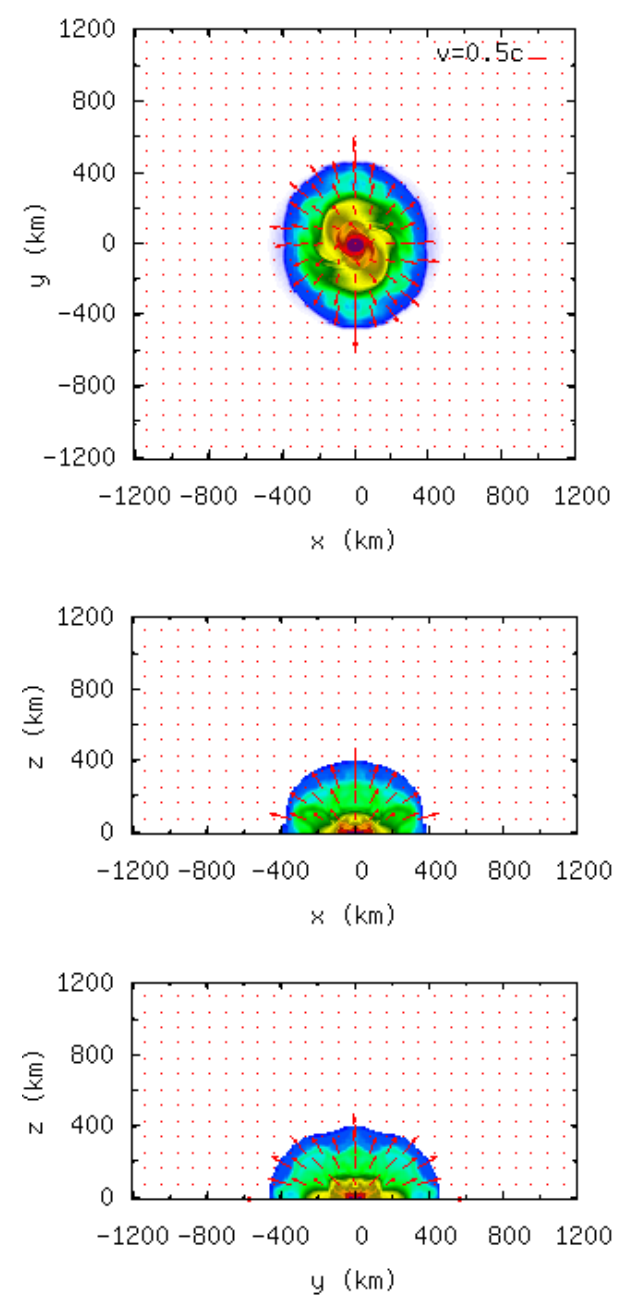

$\mathrm{t}=13.10291 \mathrm{~ms}$

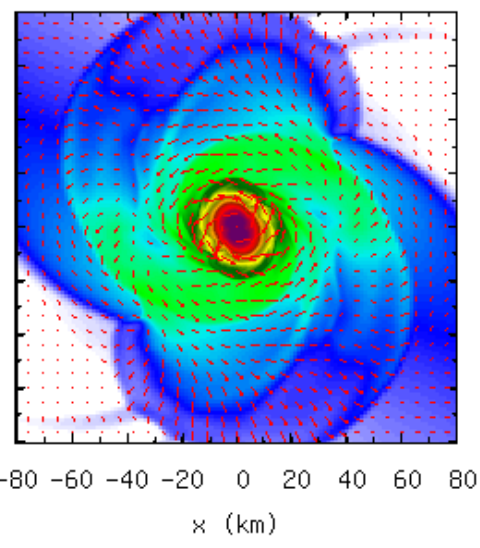

$\mathrm{t}=15.13612 \mathrm{~ms}$
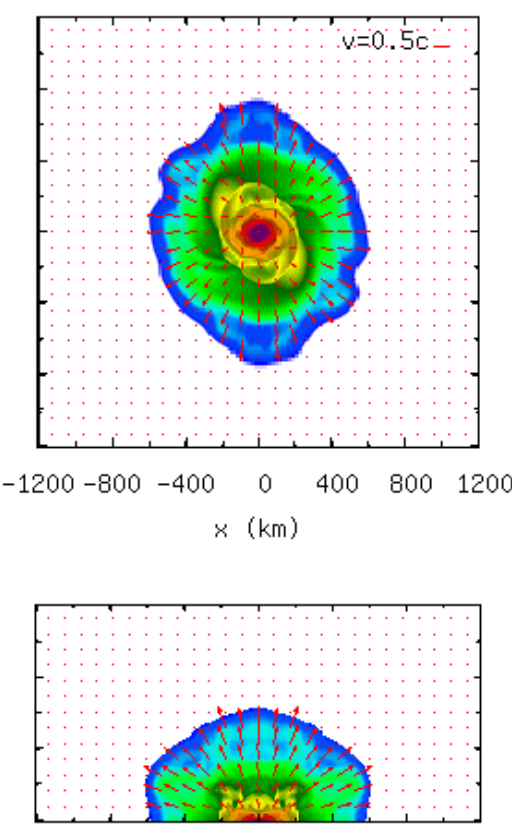

$-1200-800-400 \quad 0 \quad 400 \quad 800 \quad 1200$

$x(\mathrm{~km})$

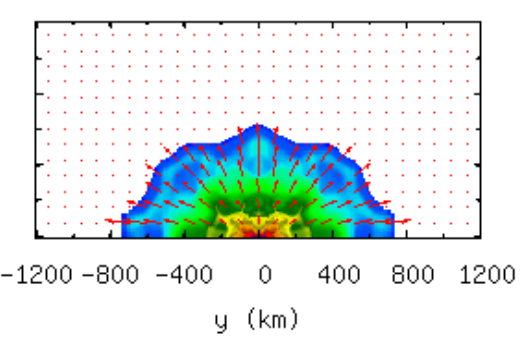

$\mathrm{t}=14.68429 \mathrm{~ms}$

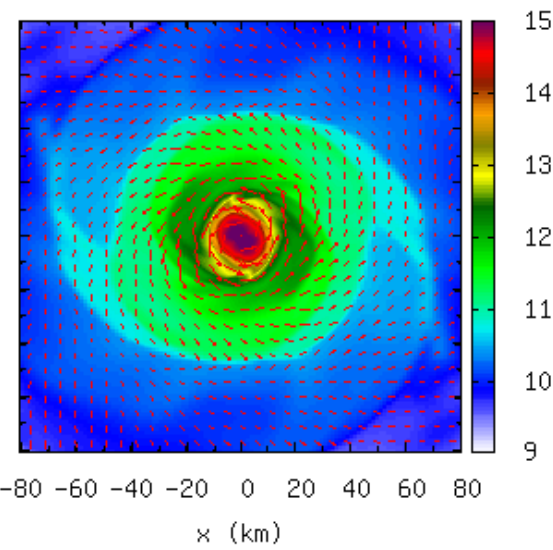

$\mathrm{t}=16.71751 \mathrm{~ms}$
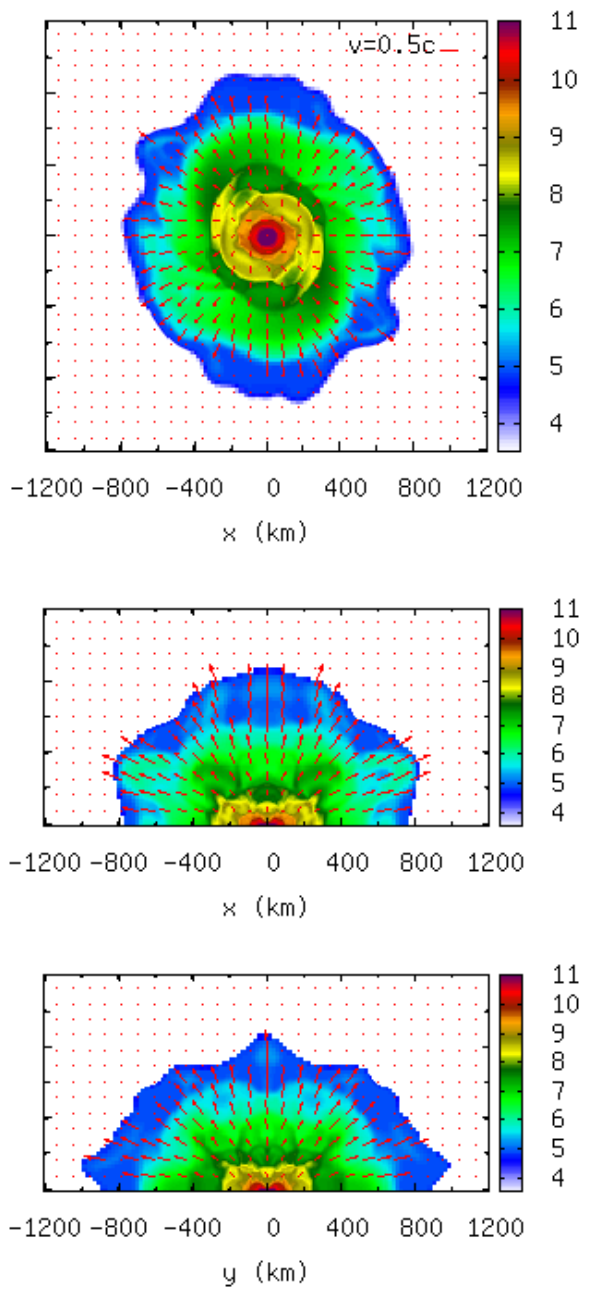

FIG. 3: Snapshots of the density profile for the merger of binary neutron stars for an equal-mass model APR4-135135. The first row shows the density profiles in the equatorial plane and in the central region, and second - fourth ones show the density profile for a wide region in the $x-y, x-z$, and $y$ - $z$ planes. $t_{\text {merge }} \approx 11.3 \mathrm{~ms}$ for this model. 


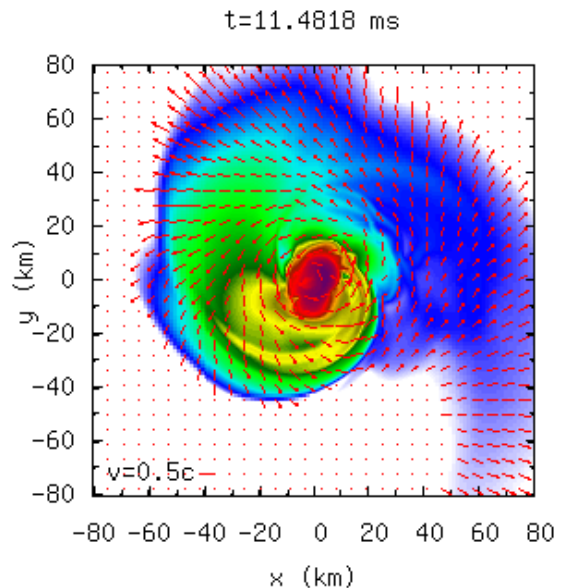

$\mathrm{t}=13.7781 \mathrm{~ms}$
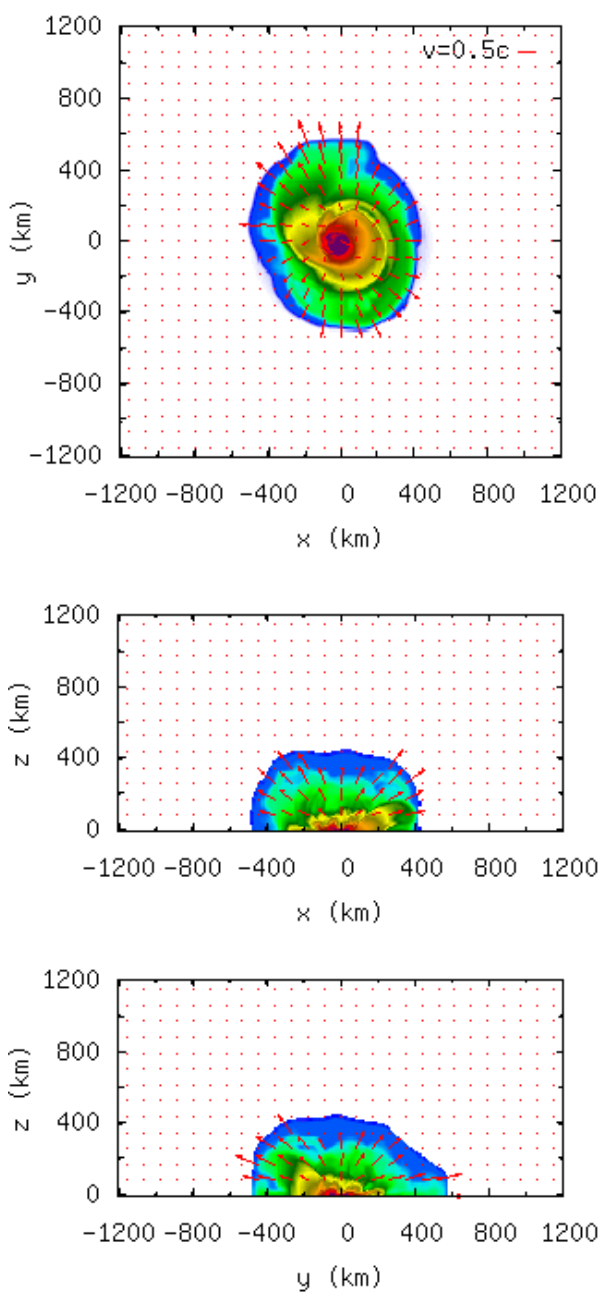

$\mathrm{t}=13.0892 \mathrm{~ms}$

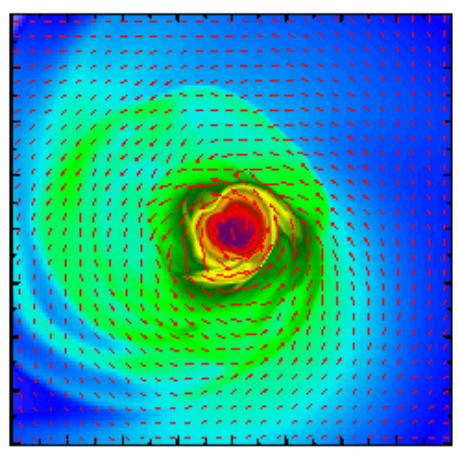

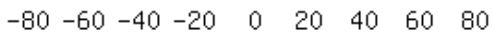

$x(\mathrm{~km})$

$\mathrm{t}=15.3856 \mathrm{~ms}$
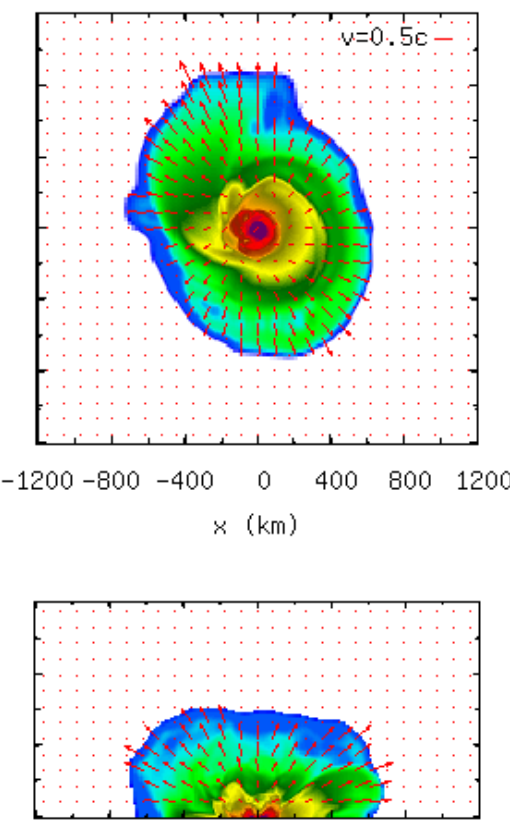

$\begin{array}{llllll}-1200-800 & -400 \quad 0 & 400 & 800 & 1200\end{array}$

$x(\mathrm{~km})$

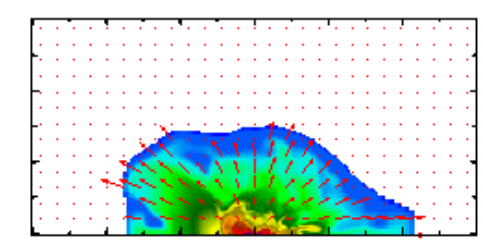

$\begin{array}{llllll}-1200-800 & -400 \quad 0 & 400 & 800 & 1200\end{array}$

y $(\mathrm{km})$ $\mathrm{t}=14.6967 \mathrm{~ms}$

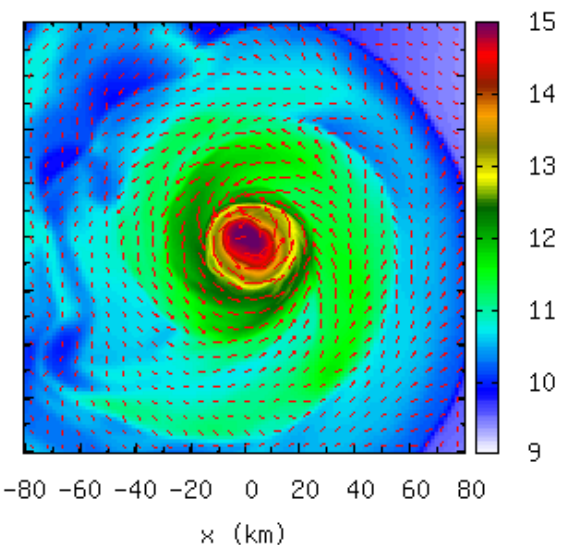

$\mathrm{t}=16.9930 \mathrm{~ms}$
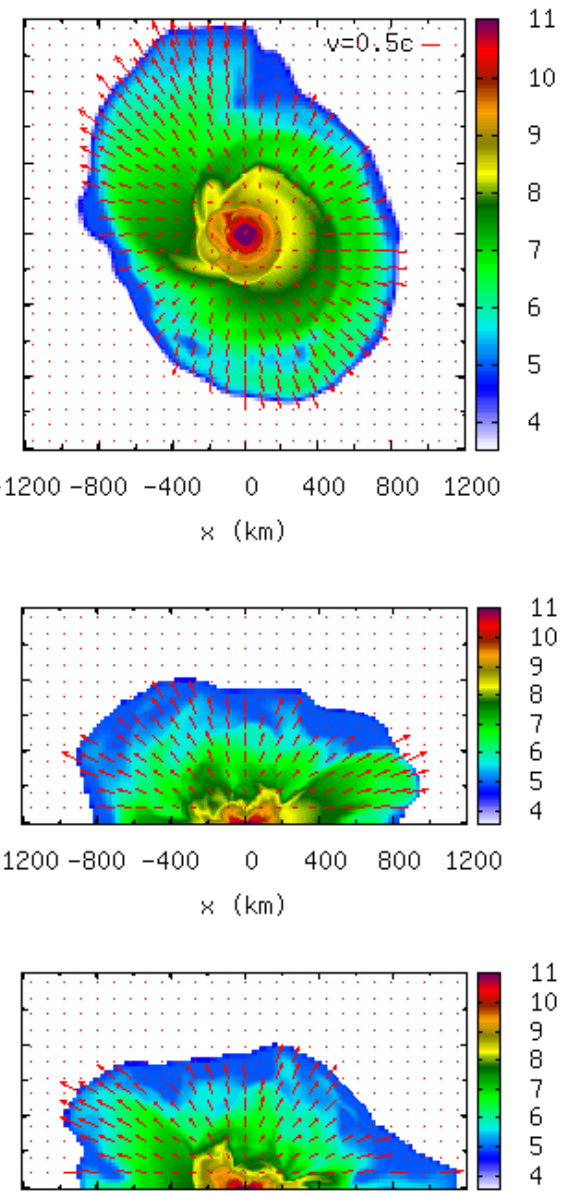

$\begin{array}{llllll}-1200-800 & -400 \quad 0 & 400 & 800 & 1200\end{array}$

FIG. 4: The same as Fig. 3, but for unequal-mass model APR4-120150. $t_{\text {merge }} \approx 10.3$ ms for this model. 


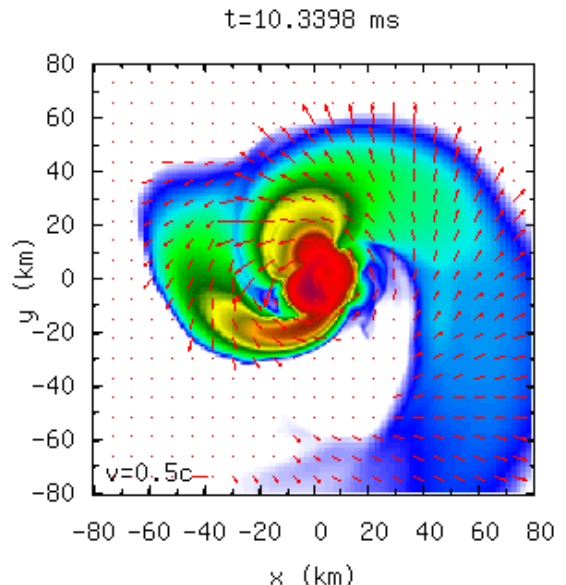

$t=12.4686 \mathrm{~ms}$
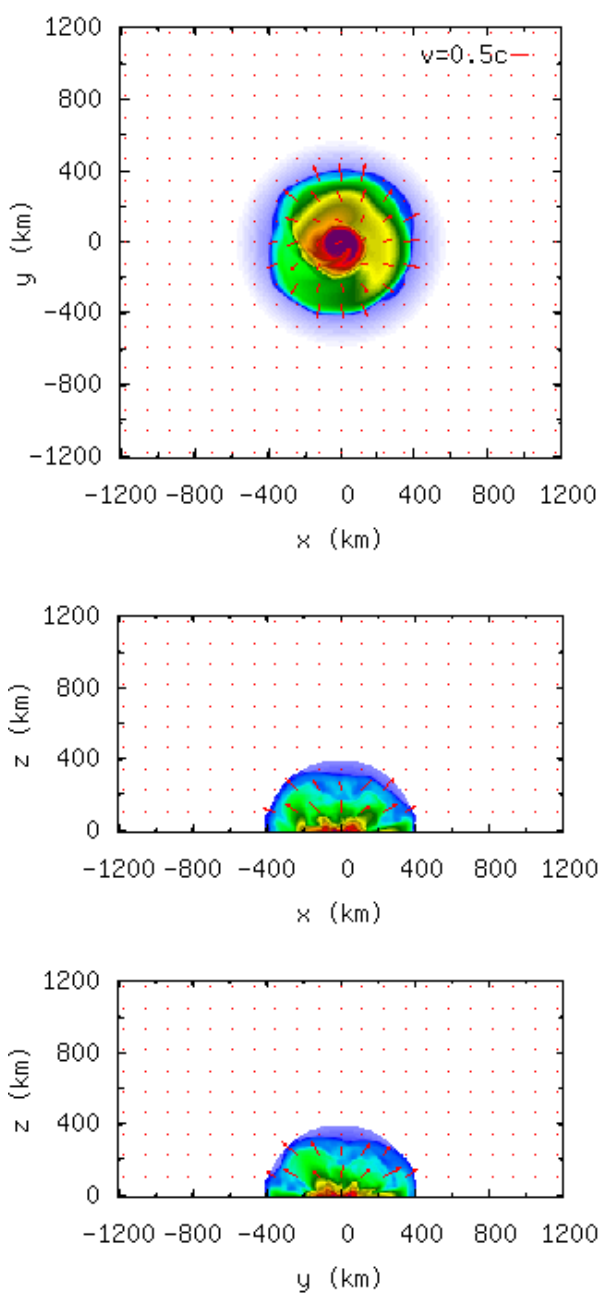

$\mathrm{t}=11.9212 \mathrm{~ms}$

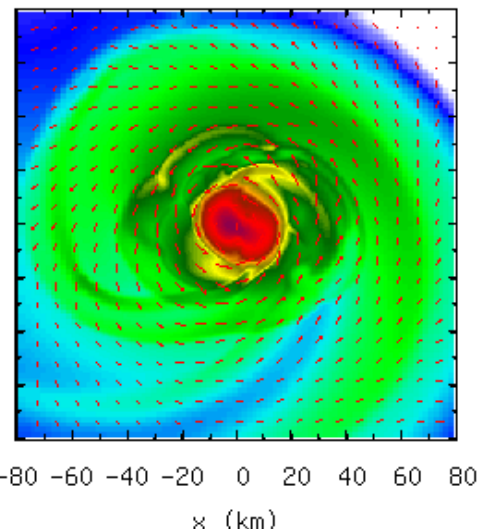

$\mathrm{t}=14.0500 \mathrm{~ms}$
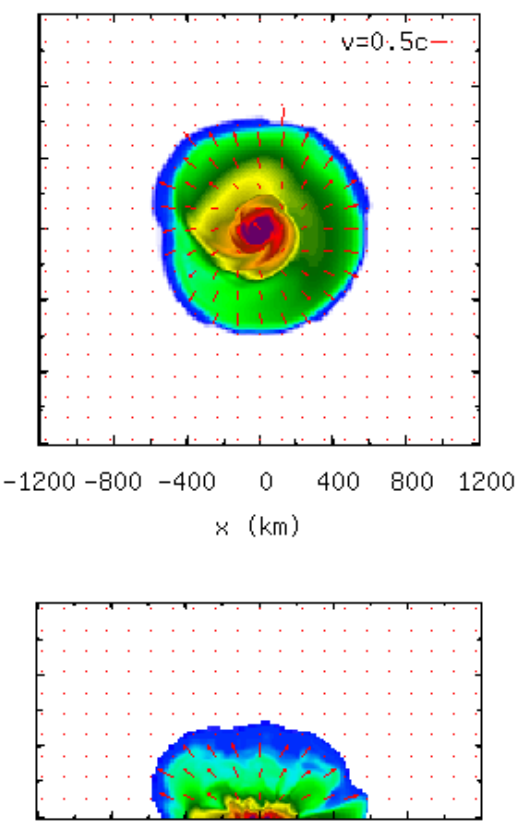

$\begin{array}{llllll}-1200-800 & -400 \quad 0 & 400 & 800 & 1200\end{array}$

$x(\mathrm{~km})$

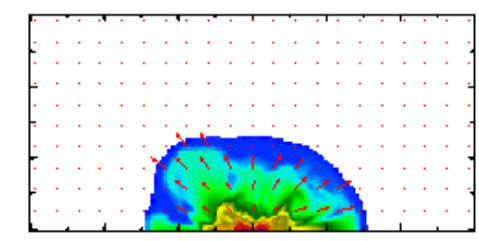

$\begin{array}{llllll}-1200-800 & -400 \quad 0 & 400 & 800 & 1200\end{array}$ y $(\mathrm{km})$

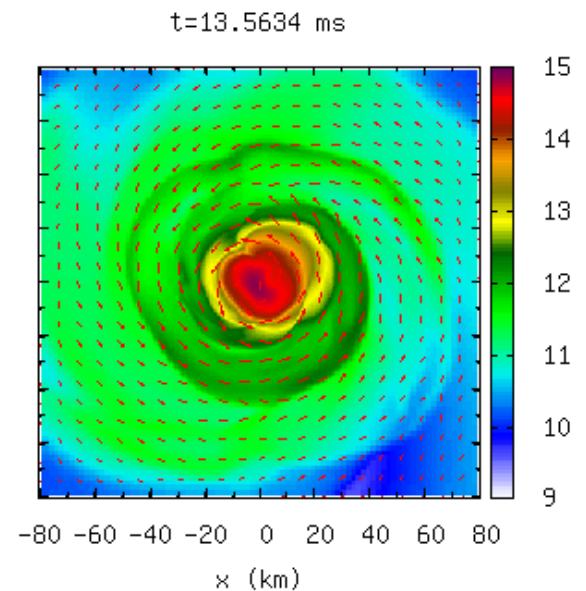

$\mathrm{t}=15.6922 \mathrm{~ms}$
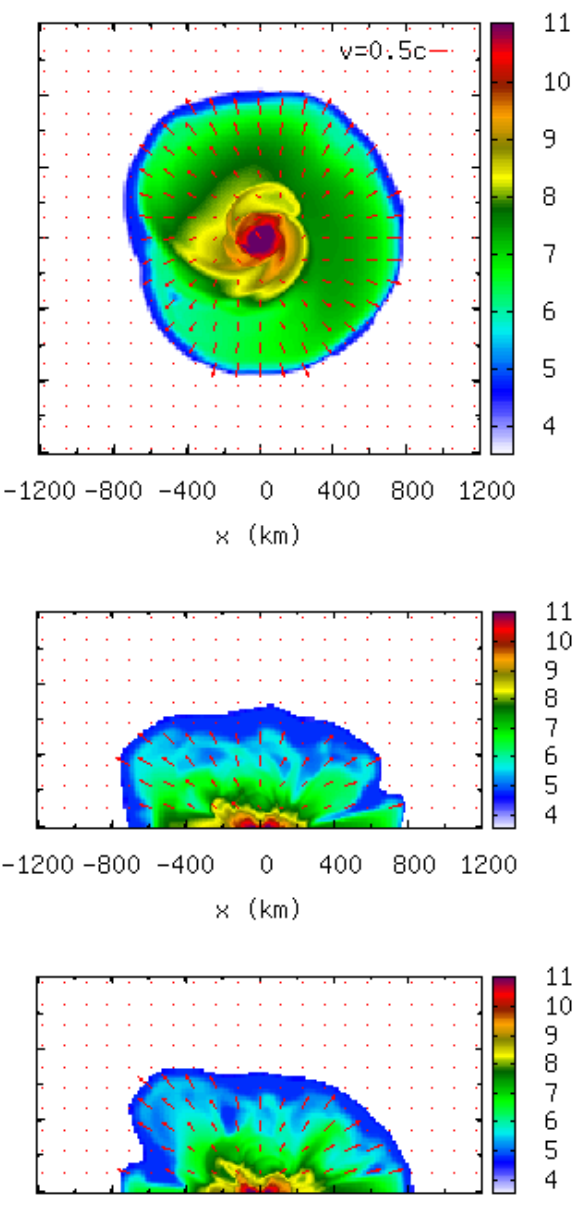

$\begin{array}{llllll}-1200-800 & -400 \quad 0 & 400 & 800 & 1200\end{array}$ y $(\mathrm{km})$

FIG. 5: The same as Fig. 4 but for models H4-120150. $t_{\text {merge }} \approx 8.8 \mathrm{~ms}$ for this model. 

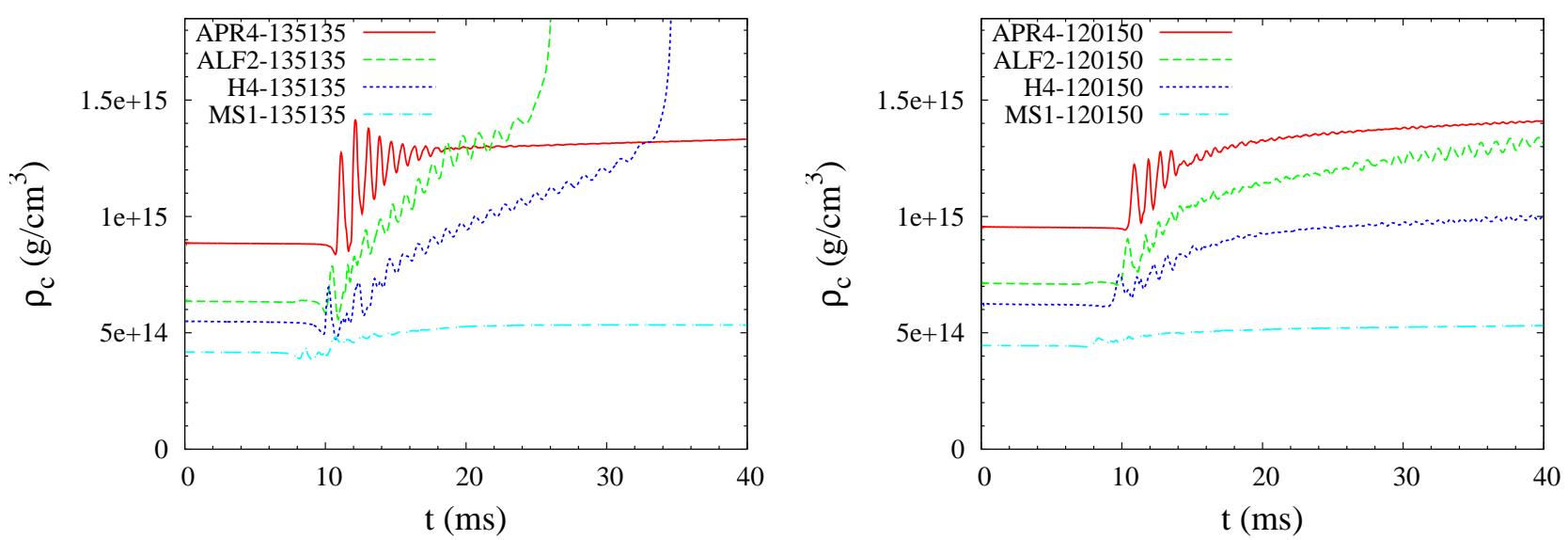

FIG. 6: The central density as a function of time for models with $m_{1}=m_{2}=1.35 M_{\odot}$ (left), and $m_{1}=1.2 M_{\odot}$ and $m_{2}=1.5 M_{\odot}$ (right). Before the merger of unequal mass binaries, the central density of heavier neutron stars are plotted. $\Gamma_{\text {th }}=1.8$ is employed for the results presented here.
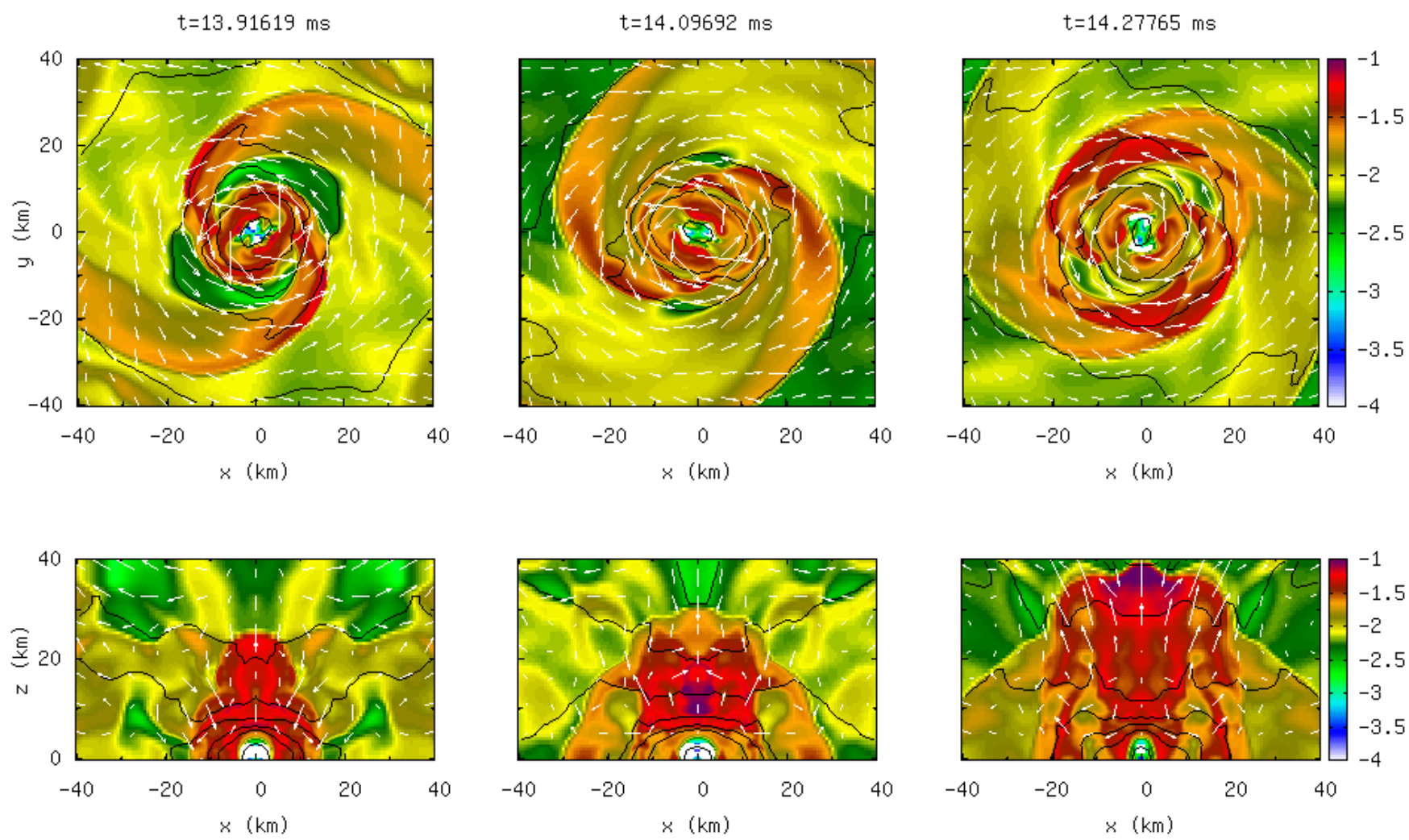

FIG. 7: Snapshots of the thermal part of the specific internal energy $\left(\varepsilon_{\mathrm{th}}\right)$ profile in the vicinity of HMNSs on the equatorial (top) and $x-z$ (bottom) planes for an equal-mass model APR4-135135. The rest-mass density contours are overplotted for every decade from $10^{15} \mathrm{~g} / \mathrm{cm}^{3}$.

Figures 3 - 5 indicate that there are two important processes for the mass ejection. The first one is the heating by shocks formed at the onset of the merger between the inner surfaces of two neutron stars. Figures 7 and 8 display snapshots of the thermal part of the specific internal energy, $\varepsilon_{\text {th }}$, in the vicinity of HMNSs for APR4-135135 and APR4-120150, respectively. These figures show clearly that hot materials with $\varepsilon_{\text {th }} \lesssim 0.1(\lesssim 100 \mathrm{MeV})$ are indeed ejected from the HMNSs, in particular, to bidirectional regions on the equatorial plane and to the polar region. This suggests that the shock heating works efficiently to eject materials from the HMNSs. This occurs 

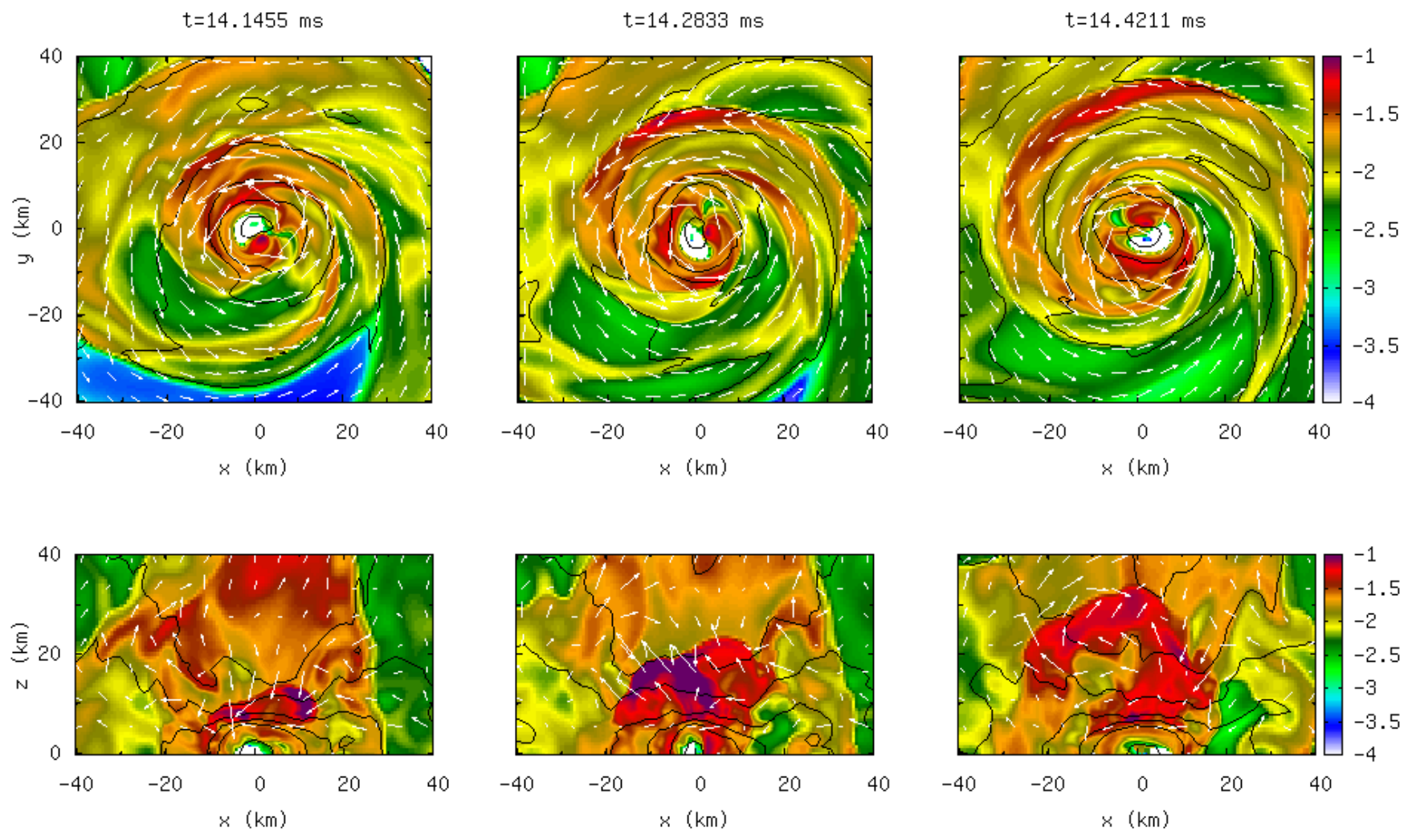

FIG. 8: The same as Fig. 8, but for an unequal-mass model APR4-120150.

in an outstanding manner in particular for the equal-mass (and only slightly asymmetric) binaries. The heated-up material is pushed outwards by the thermal pressure generated by the shock approximately in the plane parallel to the (rotating) shock surface. Subsequently, it expands outwards with rotation, and eventually forms hot spiral arms around the HMNS. This component subsequently gains angular momentum (and hence kinetic energy) due to the torque exerted by the HMNS of a nonaxisymmetric configuration, and a fraction of the material eventually gains the kinetic energy that is large enough for it to escape from the system. This effect plays a primary role for the early mass ejection that occurs in the first a few ms after the onset of the merger.

A stronger shock appears to play basically a positive role for increasing the amount of the ejected material, because the amount of the heated-up material can be more, and as a result, the materials in the spiral arm and ejected fraction increase. A stronger shock is formed for softer EOSs or for binaries composed of more compact neutron stars (e.g., APR4 in the context of canonical-mass neutron stars). The reason is that neutron stars for such an EOS can achieve a more compact state (cf. Fig. 6) and at the merger, the collision velocity of two neutron stars is larger (the minimum separation between two stars is smaller). This point will be in more detail described in Sec. IV A 3. A strong shock could be also formed for bi- naries with the total mass close to the critical value for the collapse to a black hole even for stiff EOSs, because a highly compressed state is realized by the strong gravity.

The shocks are also formed continuously in the outer part of the HMNS during its evolution through the interaction with spiral arms formed in its envelope due to a torque exerted by the HMNS (see below). This effect plays an important role in a relatively longer-term mass ejection with the duration $\sim 10-20 \mathrm{~ms}$.

The secondly important process for the mass ejection is a hydrodynamic interaction induced by the HMNS of a nonaxisymmetric configuration that exerts the torque to the surrounding material and transports the angular momentum outwards. Since it is rapidly rotating, the HMNS works as an efficient torque supplier. Our simulations show that this process is important in particular in the early phase of the merger: For the nearly equal-mass binaries, a fraction of the material that spreads outwards by the shock formed at the merger subsequently gains angular momentum from the HMNS and eventually obtains kinetic energy large enough to escape from the system; for sufficiently asymmetric binaries (for small values of $q$ ), a less-massive neutron star is tidally elongated during the early phase of the merger, a fraction of its material forms spiral arms, and it subsequently gains angular momentum from the HMNS enough to escape from the system. In the early mass ejection caused by the torque 
exerted by the HMNS, the material is primarily ejected in the direction near the equatorial plane, and the typical velocity of the escaping material in this early stage is quite high $\sim 0.5-0.8 c$ (follow the locations of the head of the ejected materials in Figs. 3-50. The maximum velocity is larger for the EOS that yields smaller-radius neutron stars; for APR4, it is $\sim 0.8 c$ and for MS1, it is $\sim 0.5 c$. This also depends on the mass ratio for models with a large neutron-star radius (for models of $\mathrm{H} 4$ and MS1).

In the later phase, the mass ejection appears to occur by the combination of the shock heating and by the torque exerted by the HMNS. As mentioned already, the continuous shock heating occurs in the envelope of the HMNS in the presence of spiral arms. Due to this, a fraction of the material gains large kinetic energy. In addition, the material in the outer region gains angular momentum by the torque exerted by the HMNS. These two effects give a fraction of the material the escape velocity. By this process, the material is gradually ejected from the system in a quasispherical manner; the anisotropy of the configuration of the ejected material is not as large as that of the material ejected in the early stage. This indicates that the shock heating plays a relatively important role. The average velocity of the escaping material in this process is sub-relativistic $\sim 0.15-0.25 c$ (see Table IV).

In the mass ejection process, these two nonlinearly coupled effects (shock heating and torque exerted by the HMNS) play a substantial role. As a result, the amount of the ejected material depends on the EOS, the total mass of the system, and the mass ratio in a nonlinear manner. Thus, a small change (associated, e.g., with the grid resolution, the initial orbital separation, configuration of the atmosphere, and presence or absence of the $\pi$ symmetry for equal-mass binaries) results in the change in the rest mass and kinetic energy of the ejected material; this fluctuation is in general small, $\sim 10-20 \%$, for unequal-mass binaries for which the torque plays a primary role (see Appendix A). For the equal-mass case, the convergence is poor because a strong shock often occurs at the merger and plays a primary role in the mass ejection. The possible reason for this poor convergence is that shocks are always computed by the first-order accuracy in the spatial grid resolution, and hence, the accuracy is low and in addition, the ejected mass is a tiny part of the entire system. A random error for the entire system computed with a low accuracy significantly (and randomly) affects a tiny part (i.e., the ejected material), resulting in the poor convergence. (We note that for global quantities, the convergence is usually good.) For some models (such as ALF2-135135 and MS1-135135), the ejected mass increases steeply with the grid resolution, and for such cases, the results in this paper might give the lower bound.

In the following subsections, we describe the properties of the ejected material in more detail.

\section{Gravitational waves}

First of all, we summarize the properties of gravitational waves emitted by the HMNS, because its gravitational-wave frequency, which is determined by the spin of the HMNS, has a correlation with the amount of the ejected material

As mentioned already, HMNSs exert the torque to its surrounding material. The efficiency of the angular momentum transport is higher, in general, for the faster rotating and more compact HMNS. Associated with this property, the frequency of gravitational waves and the efficiency of the angular momentum transport are expected to be closely related. The characteristic spin frequency for these deformed HMNSs can be determined from gravitational waves emitted by them. Figure 9 displays gravitational waves and their frequency as functions of time for eight models with mass $\left(m_{1}, m_{2}\right)=\left(1.3 M_{\odot}, 1.4 M_{\odot}\right)$ and $\left(m_{1}, m_{2}\right)=\left(1.2 M_{\odot}, 1.5 M_{\odot}\right)$ and with four EOSs. These plots show that quasiperiodic gravitational waves are emitted by the HMNSs for all the models. Namely, the gravitational-wave frequency does not change significantly during the evolution of the HMNSs. However, the frequency is not constant exactly and actually varies with time. This is natural because (i) the HMNSs quasiradially oscillate with time in their early stage of the evolution, and (ii) the HMNSs lose the energy and angular momentum due to the gravitational-wave emission and hydrodynamic angular momentum transport process, and hence, their configuration evolves. These two effects result in the variation in the characteristic spin velocity and frequency of gravitational waves. The degree of the variation in the frequency of gravitational waves is larger (a) for the HMNS with the EOS that yields a compact neutron star (we often call such an EOS soft EOS in this paper), and (b) for the HMNS for which the mass is close to the critical value to the collapse to a black hole; see, e.g., the gravitational-wave frequency for model ALF2-130140. The case (a) is due to the fact that at the merger, the central density significantly increases in the soft EOSs, resulting in subsequent high-amplitude oscillations. The case (b) is due to the fact that for such a HMNS, a small change in the spin velocity results in a large change in the central density.

Figure 10 plots the Fourier spectra for gravitational waves shown in Fig. 9. This shows that there are peaks for a high-frequency band $2 \mathrm{kHz} \lesssim f \lesssim 4 \mathrm{kHz}$ irrespective of models. For a "soft" EOS that yields a compact neutron star for the canonical mass, the peak frequency is higher (e.g., for the spectra of APR4, the peak frequency is the highest among the four EOSs), and a certain correlation exists between the peak frequency and stellar radius 49. The peak frequency is approximately associated with the typical frequency of quasiperiodic oscillation of gravitational waves found in Fig. 9. However, as already mentioned, the (nonaxisymmetric) oscillation frequencies of the HMNSs vary during the evolution due to a quasiradial oscillation and the back reaction 

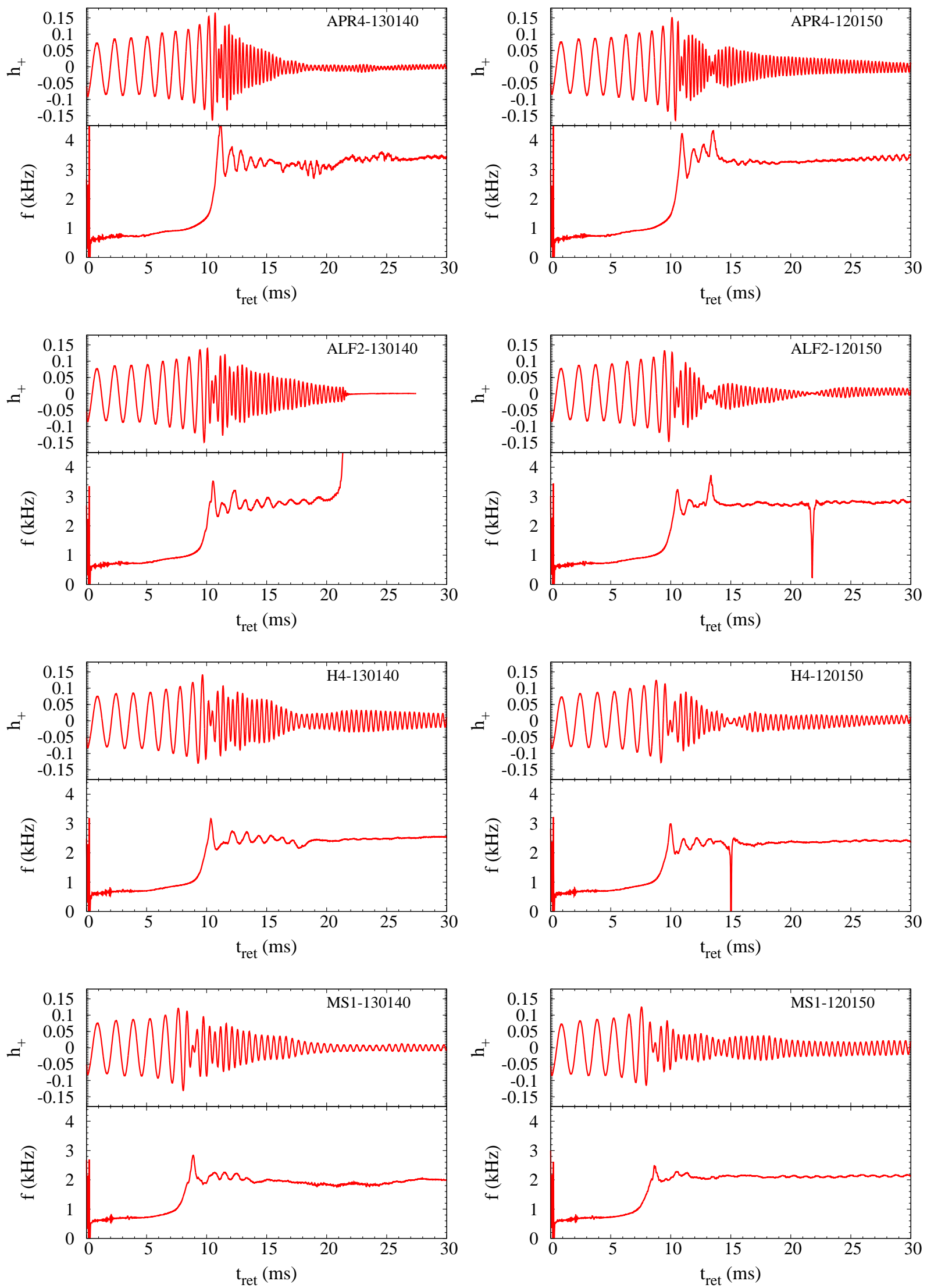

FIG. 9: Gravitational waves $\left(h_{+} D / m\right)$ and the frequency of gravitational waves $f$ as functions of time for models APR4-130140 (top left), APR4-120150 (top right), ALF2-130140 (second top left), ALF2-120150 (second top right), H4-130140 (third left), H4-120150 (third right), MS1-130140 (bottom left), and MS1-120150 (bottom right). For ALF2-130140, a black hole is formed at $11 \mathrm{~ms}$ after the onset of the merger, and ringdown gravitational waves are emitted in the final phase. For all the panels, the vertical axis shows the non-dimensional amplitude, $h_{+} D / m$, with $D$ being the distance to the source. 
TABLE IV: Summary of numerical results. The remnant, the total rest mass, $M_{* \text { esc }}$, the kinetic energy, $T_{* \text { esc }}$, the $R$ and $Z$ components of the average velocity of escaping material, $\bar{V}_{\mathrm{esc}}^{R}$ and $\bar{V}_{\mathrm{esc}}^{Z}$, of the ejected material, and characteristic frequencies of gravitational waves emitted by HMNSs for 5 and $10 \mathrm{~ms}$ time integration after the formation of the HMNSs. The total rest mass, kinetic energy, and average velocity are measured at $\approx 10 \mathrm{~ms}$ after the onset of the merger. The dispersion of $f_{\text {ave }}$ shown here is $\sigma_{f}$. BH denotes black hole. The remnant is judged at $\approx 30 \mathrm{~ms}$ after the onset of the merger. All the results shown are those in the run with $N=60$ and our standard setting of atmosphere. The rest mass and kinetic energy of the ejected material have the uncertainty of order 10\%. The approximate lifetime of HMNSs for APR4-130150, APR4-140140, ALF2-140140, ALF2-130140, ALF2-135135, H4-130150, H4-140140, H4-135135 ( $\left.\Gamma_{\text {th }}=1.6\right)$ ), and H4-135135 $\left.\left(\Gamma_{\text {th }}=1.8\right)\right)$ is $\sim 30,30,5,10,15,20,10,15$, $25 \mathrm{~ms}$ for $N=60$, respectively.

\begin{tabular}{|c|c|c|c|c|c|c|c|c|}
\hline Model & $\Gamma_{\text {th }}$ & Remnant & $M_{* \mathrm{esc}}\left(10^{-3} M_{\odot}\right)$ & $T_{* \mathrm{esc}}\left(10^{50} \mathrm{ergs}\right)$ & $\bar{V}_{\mathrm{esc}}^{R} / c$ & $\bar{V}_{\mathrm{esc}}^{Z} / c$ & $f_{\text {ave }, 5 \mathrm{~ms}}(\mathrm{kHz})$ & $f_{\text {ave }, 10 \mathrm{~ms}}(\mathrm{kHz})$ \\
\hline 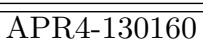 & 1.8 & $\mathrm{BH}$ & 2.0 & 1.5 & 0.24 & 0.08 & - & - \\
\hline APR4-140150 & 1.8 & $\mathrm{BH}$ & 0.6 & 0.9 & 0.35 & 0.12 & - & - \\
\hline APR4-145145 & 1.8 & $\mathrm{BH}$ & 0.1 & $<0.1$ & 0.29 & 0.13 & - & - \\
\hline APR4-130150 & 1.8 & $\mathrm{HMNS} \rightarrow \mathrm{BH}$ & 12 & 8.5 & 0.23 & 0.12 & $3.48 \pm 0.47$ & $3.46 \pm 0.37$ \\
\hline APR4-140140 & 1.8 & $\mathrm{HMNS} \rightarrow \mathrm{BH}$ & 14 & 10 & 0.22 & 0.15 & $3.53 \pm 0.52$ & $3.52 \pm 0.48$ \\
\hline APR4-120150 & 1.6 & HMNS & 9 & 5 & 0.20 & 0.10 & $3.47 \pm 0.30$ & $3.44 \pm 0.27$ \\
\hline APR4-120150 & 1.8 & HMNS & 8 & 5.5 & 0.23 & 0.11 & $3.44 \pm 0.30$ & $3.41 \pm 0.24$ \\
\hline APR4-120150 & 2.0 & HMNS & 7.5 & 5.5 & 0.24 & 0.12 & $3.32 \pm 0.32$ & $3.27 \pm 0.26$ \\
\hline APR4-125145 & 1.8 & HMNS & 7 & 4.5 & 0.22 & 0.11 & $3.36 \pm 0.31$ & $3.31 \pm 0.25$ \\
\hline APR4-130140 & 1.8 & HMNS & 8 & 5 & 0.19 & 0.12 & $3.30 \pm 0.29$ & $3.27 \pm 0.28$ \\
\hline APR4-135135 & 1.6 & HMNS & 11 & 6 & 0.19 & 0.13 & $3.46 \pm 0.42$ & $3.45 \pm 0.37$ \\
\hline APR4-135135 & 1.8 & HMNS & 7 & 4 & 0.19 & 0.12 & $3.31 \pm 0.35$ & $3.31 \pm 0.32$ \\
\hline APR4-135135 & 2.0 & HMNS & 5 & 3 & 0.19 & 0.13 & $3.35 \pm 0.39$ & $3.33 \pm 0.33$ \\
\hline APR4-120140 & 1.8 & HMNS & 3 & 2 & 0.21 & 0.12 & $3.15 \pm 0.21$ & $3.13 \pm 0.19$ \\
\hline APR4-125135 & 1.8 & HMNS & 5 & 3 & 0.18 & 0.10 & $3.22 \pm 0.25$ & $3.19 \pm 0.24$ \\
\hline APR4-130130 & 1.8 & HMNS & 2 & 1 & 0.19 & 0.10 & $3.22 \pm 0.28$ & $3.19 \pm 0.26$ \\
\hline ALF2-140140 & 1.8 & $\mathrm{HMNS} \rightarrow \mathrm{BH}$ & 2.5 & 1.5 & 0.21 & 0.13 & $2.93 \pm 0.42$ & - \\
\hline ALF2-120150 & 1.8 & HMNS & 5.5 & 3 & 0.21 & 0.10 & $2.70 \pm 0.19$ & $2.71 \pm 0.16$ \\
\hline ALF2-125145 & 1.8 & HMNS & 3 & 1.5 & 0.20 & 0.10 & $2.66 \pm 0.14$ & $2.66 \pm 0.13$ \\
\hline ALF2-130140 & 1.8 & $\mathrm{HMNS} \rightarrow \mathrm{BH}$ & 1.5 & 0.8 & 0.16 & 0.11 & $2.73 \pm 0.19$ & $2.75 \pm 0.17$ \\
\hline ALF2-135135 & 1.8 & $\mathrm{HMNS} \rightarrow \mathrm{BH}$ & 2.5 & 1.5 & 0.22 & 0.12 & $2.75 \pm 0.18$ & $2.76 \pm 0.16$ \\
\hline ALF2-130130 & 1.8 & HMNS & 2 & 1.0 & 0.19 & 0.10 & $2.58 \pm 0.18$ & $2.56 \pm 0.16$ \\
\hline H4-130150 & 1.8 & $\mathrm{HMNS} \rightarrow \mathrm{BH}$ & 3 & 2 & 0.19 & 0.10 & $2.44 \pm 0.17$ & $2.45 \pm 0.15$ \\
\hline H4-140140 & 1.8 & $\mathrm{HMNS} \rightarrow \mathrm{BH}$ & 0.3 & 0.2 & 0.17 & 0.13 & $2.63 \pm 0.23$ & $2.77 \pm 0.41$ \\
\hline H4-120150 & 1.6 & HMNS & 4.5 & 2 & 0.19 & 0.10 & $2.28 \pm 0.16$ & $2.29 \pm 0.14$ \\
\hline H4-120150 & 1.8 & HMNS & 3.5 & 2 & 0.21 & 0.09 & $2.30 \pm 0.18$ & $2.31 \pm 0.15$ \\
\hline H4-120150 & 2.0 & HMNS & 4 & 2 & 0.21 & 0.09 & $2.24 \pm 0.15$ & $2.23 \pm 0.14$ \\
\hline H4-125145 & 1.8 & HMNS & 2 & 1.5 & 0.19 & 0.10 & $2.41 \pm 0.15$ & $2.41 \pm 0.13$ \\
\hline H4-130140 & 1.8 & HMNS & 0.7 & 0.4 & 0.18 & 0.10 & $2.42 \pm 0.17$ & $2.42 \pm 0.15$ \\
\hline H4-135135 & 1.6 & $\mathrm{HMNS} \rightarrow \mathrm{BH}$ & 0.7 & 0.4 & 0.21 & 0.11 & $2.49 \pm 0.19$ & $2.54 \pm 0.16$ \\
\hline H4-135135 & 1.8 & $\mathrm{HMNS} \rightarrow \mathrm{BH}$ & 0.5 & 0.2 & 0.19 & 0.11 & $2.44 \pm 0.20$ & $2.48 \pm 0.16$ \\
\hline H4-135135 & 2.0 & HMNS & 0.4 & 0.2 & 0.20 & 0.10 & $2.39 \pm 0.21$ & $2.43 \pm 0.17$ \\
\hline H4-120140 & 1.8 & HMNS & 2.5 & 1 & 0.19 & 0.10 & $2.30 \pm 0.15$ & $2.30 \pm 0.14$ \\
\hline H4-125135 & 1.8 & HMNS & 0.6 & 0.3 & 0.18 & 0.10 & $2.29 \pm 0.17$ & $2.27 \pm 0.14$ \\
\hline H4-130130 & 1.8 & HMNS & 0.3 & 0.1 & 0.16 & 0.10 & $2.35 \pm 0.18$ & $2.38 \pm 0.14$ \\
\hline MS1-140140 & 1.8 & MNS & 0.6 & 0.2 & 0.13 & 0.09 & $2.09 \pm 0.14$ & $2.06 \pm 0.12$ \\
\hline MS1-120150 & 1.8 & MNS & 3.5 & 1.5 & 0.19 & 0.10 & $2.08 \pm 0.11$ & $2.09 \pm 0.09$ \\
\hline MS1-125145 & 1.8 & MNS & 1.5 & 0.8 & 0.19 & 0.11 & $2.02 \pm 0.14$ & $1.99 \pm 0.15$ \\
\hline MS1-130140 & 1.8 & MNS & 0.6 & 0.2 & 0.17 & 0.09 & $2.05 \pm 0.14$ & $2.02 \pm 0.13$ \\
\hline MS1-135135 & 1.8 & MNS & 1.5 & 0.6 & 0.14 & 0.08 & $1.98 \pm 0.18$ & $1.95 \pm 0.16$ \\
\hline MS1-130130 & 1.8 & MNS & 1.5 & 0.5 & 0.15 & 0.08 & $1.93 \pm 0.19$ & $1.90 \pm 0.17$ \\
\hline
\end{tabular}

due to the gravitational-wave emission and angular momentum transport process, and hence, the peak frequencies change with time, resulting in the broadening of the peak or appearance of the multi peaks. Therefore, it is not a very good idea to determine the characteristic frequency from the peak of the Fourier spectrum. Rather, the Fourier spectrum might provide an inaccurate message when we determine the characteristic oscillation fre- quency. Thus, we determine the average frequency from the results of the frequency shown in Fig. 9 in terms of Eq. (14) with the dispersion determined by Eq. (15). Here, the time integration is performed for 5 and $10 \mathrm{~ms}$ after the formation of the HMNSs. The last two columns of Table IV list the average frequency and the dispersion determined for 5 and $10 \mathrm{~ms}$ integration. Note that the typical nonaxisymmetric oscillation frequency of the 

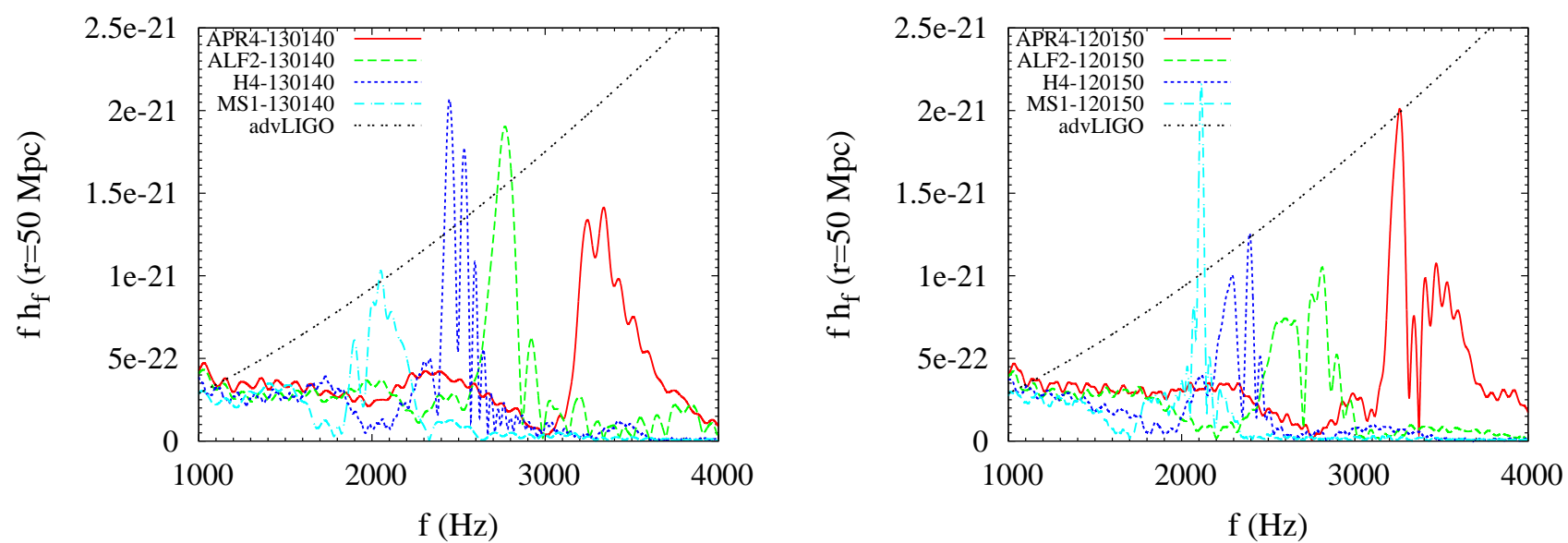

FIG. 10: Fourier spectra of gravitational waves for the results shown in Fig. 9 . The amplitude is shown for the hypothetical event at a distance of $50 \mathrm{Mpc}$ along the direction perpendicular to the orbital plane (the most optimistic direction). The black dot-dot curve is the noise spectrum of the advanced LIGO with an optimistic configuration for the detection of high-frequency gravitational waves (see https://dcc.ligo.org/cgi-bin/DocDB/ShowDocument?docid=2974).

HMNSs is half as large as the values listed in Table IV because the listed ones are the gravitational-wave frequencies.

The value of the oscillation frequency for a given mass of the HMNS depends primarily on its radius, i.e., a stiffness of the EOS. For the EOS that yields smallradius neutron stars ("soft" EOS), the oscillation frequency and peak frequency of gravitational waves are higher, because the spin angular velocity of the HMNS is close to the Kepler velocity, and thus, the oscillation and peak frequencies are qualitatively proportional to $\left(M_{\mathrm{HMNS}} / R_{\mathrm{HMNS}}^{3}\right)^{1 / 2}$ where $M_{\mathrm{HMNS}}$ and $R_{\mathrm{HMNS}}$ denote the typical mass and radius of a HMNS. The oscillation frequency depends also weakly on the value of $\Gamma_{\mathrm{th}}$ : For the smaller value of it, the frequency is slightly higher for many cases, because the effect of shock heating is weaker, and the HMNS becomes more compact.

For a larger spin of the nonaxisymmetric HMNS, the material surrounding the HMNS can receive a torque with a higher efficiency. This suggests that for the merger of a binary neutron star composed of smaller-radius neutron stars, the amount of the ejected material could be larger. As shown in Sec. IV A3, this is indeed the case (in particular for unequal-mass models), as long as the models in this paper are concerned.

Table IV] as well as Fig. 10 also show that the magnitude of the dispersion, $\sigma_{f}$, is not negligible. For APR4 for which the neutron-star radius is rather small and the amplitude of a quasiradial oscillation induced at the formation of the HMNSs is rather large, the magnitude of the dispersion is $0.2-0.5 \mathrm{kHz}$; for $m=2.7 M_{\odot}$, the typical value is $0.3-0.4 \mathrm{kHz}$. For other EOSs, the dispersion is relatively small. However, it is still $0.1-0.2 \mathrm{kHz}$. Thus, we conclude that the characteristic frequency of gravitational waves from HMNSs varies with time in general.

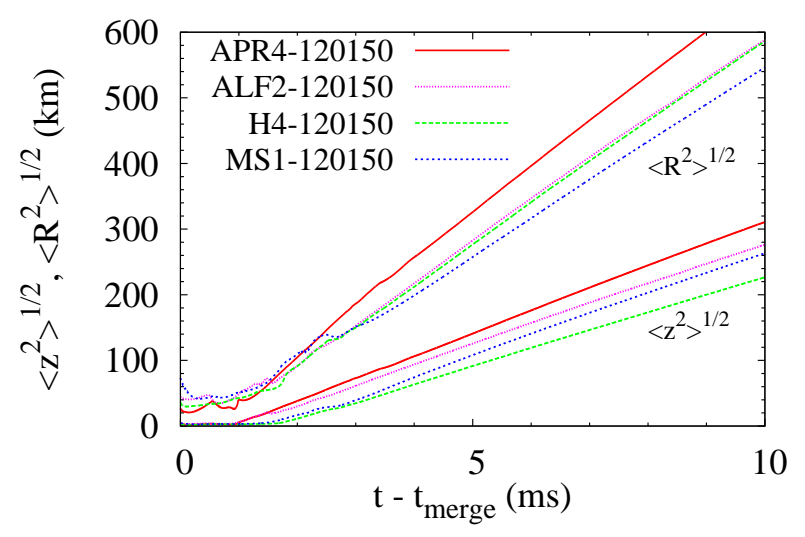

FIG. 11: $\quad \bar{R}\left(=\left\langle R^{2}\right\rangle^{1 / 2}\right)$ and $\bar{Z}\left(=\left\langle z^{2}\right\rangle^{1 / 2}\right)$ as functions of time for APR4-120150, ALF2-120150, H4-120150, and MS1120150 .

\section{Average velocity of the ejected material}

Figure 11 plots $\bar{R}$ and $\bar{Z}$ as functions of time for APR4120150, ALF2-120150, H4-120150, and MS1-120150. Note that $d \bar{R} / d t$ and $d \bar{Z} / d t$ may be considered as an average velocity of the ejected material in the cylindrical and vertical directions, respectively, and that a similar result is found for other choices of mass. This shows that the material ejected expands with an approximately constant sub-relativistic velocity $\sim 0.15-0.25 c$ for $t-t_{\text {merger }} \gtrsim 2 \mathrm{~ms}$ in the cylindrical direction and the velocity in the vertical direction is $0.4-0.5$ times as large as that in the cylindrical direction. This suggests that the vertical thickness angle of the ejected material, $\theta_{0}$, is $\sim 40-50^{\circ}$. Namely, the ejected material expands in a moderately anisotropic manner. Note that the velocity in the cylindrical direction is primarily caused by the 
torque exerted by the HMNS, while the velocity in the vertical direction is primarily caused by the shock heating. This implies that both effects play an important role.

The velocity in the later phase, $t-t_{\text {merge }} \gtrsim 3 \mathrm{~ms}$, is larger for APR4 than that for other EOSs employed in this paper. This is due to the fact that with APR4, a more compact state is realized in the HMNS, and hence, (i) a strong shock associated with the compression by a strong gravity and a subsequent large-amplitude oscillation (cf. Fig. 6) occurs, resulting in an efficient mass ejection, and (ii) the HMNS strongly exerts the torque to its surrounding material. For APR4, a relatively dense atmosphere surrounding the HMNS is formed not only in the vicinity of the equatorial plane but also in the vertical direction (compare Figs. 4 and 5). This also reflects the fact that a strong shock heating occurs with this EOS (see Figs. 7 and 8 ).

\section{Dependence on EOS}

Figure 12 plots the total rest mass and kinetic energy of the material ejected from the HMNSs as functions of $t-t_{\text {merge }}$ for several models; for the left and right panels, the masses of two neutron stars are $\left(1.3 M_{\odot}, 1.4 M_{\odot}\right)$ and $\left(1.2 M_{\odot}, 1.5 M_{\odot}\right)$, respectively, with the total mass $2.7 M_{\odot}$, while four EOSs are chosen. This shows that the rest mass and kinetic energy of the ejected material depend strongly on the EOS. The primary reason is that the compactness of the HMNS depends strongly on the EOS. For APR4 and ALF2, neutron stars of canonical masses $1.2-1.5 M_{\odot}$ have a relatively small radius (cf. Table I). This implies that the merger sets in at a compact orbit, and the formed HMNS is more compact than that formed in stiffer EOSs that yield large-radius neutron stars.

A high compactness of a HMNS affects the properties of the material ejected from it in the following two ways. First, the HMNS is more rapidly rotating, and hence, it exerts the torque, caused by its nonaxisymmetric configuration and rapid rotation, to the material in the outer region more efficiently than a less compact HMNS. As a result of this effect, a fraction of the material that gains the kinetic energy large enough to escape from the system is increased. In addition, during the formation of such a compact HMNS, a quasiradial oscillation with a high amplitude is often induced (see Fig. 6). This is in particular the case for APR4 in which the EOS becomes stiff for a high-density region although it is rather soft for the density of canonical-mass neutron stars. This quasiradial oscillation helps the material surrounding the HMNS to obtain kinetic energy through shock heating (see section IV A 1).

A possibly important fact to be noted is that the material, which eventually escapes from the system, initially stays in the vicinity of the HMNS. Namely, this material stays in a deep gravitational potential well initially, and thus, it is trapped. For a more compact HMNS, this potential should be deeper, and hence, the material there needs to obtain more energy to escape from the HMNS. At the same time, however, such a material can gain a stronger torque and thermal energy for a longer timescale, because it is trapped for a longer duration, and as a result, the material could get more kinetic energy if the HMNS is more compact.

Namely, there are two competing effects, and it is not trivial at all which effects are more important. If the trapping effect due to the deep potential well plays a more important role, we should find the evidence that less material is ejected from more compact HMNS. However, Fig. 12 shows that the ejected rest mass is smaller for EOS with larger neutron star radii (less compact neutron star). This indicates that the trapping mechanism is less important than the effects of the quasiradial oscillation and the torque exerted from the HMNS, as long as the comparison among four model EOSs is concerned (but see Sec. IV A 5 for an evidence that this may not be always the case).

The compactness of HMNSs, and hence, the EOS of neutron stars, are well reflected in the frequency of gravitational waves emitted by the HMNS, as already described in Sec. IVA 1. For a given total mass and mass ratio of the binary system, the frequencies are higher for binaries composed of more compact neutron stars ("softer" EOS), because the formed HMNS is more compact and hence the rotational angular velocity approximately proportional to $\left(M_{\mathrm{HMNS}} / R_{\mathrm{HMNS}}^{3}\right)^{1 / 2}$ is larger. Since we found that the rest mass and kinetic energy of the ejected material are larger for the EOS that yields more compact HMNSs, these quantities and the frequency of gravitational waves should have a correlation.

Figure 13 plots the rest mass of the ejected material as a function of the characteristic frequency of gravitational waves emitted by the HMNS, $f_{\text {ave }}$, for several models. Here, $f_{\text {ave }}$ is determined by the $5 \mathrm{~ms}$ integration using Eq. (15). For this plot, the results with $\Gamma_{\text {th }}=1.8$ are adopted. Note that the typical frequency for this plot is determined primarily by the chosen EOS. This figure shows that for a given mass ratio $q$, these two quantities have a correlation; the total rest mass of the ejected material increases with the gravitational-wave frequency.

However, as already noted, there are counter examples (see Sec. IV A 5). Namely, for some cases, the shallow potential helps in enhancing the mass ejection. For such models, the correlation like that found in Fig. 13 does not hold.

\section{Dependence on $\Gamma_{\mathrm{th}}$}

The total rest mass and kinetic energy for the ejected material depend also on the value of $\Gamma_{\text {th }}$. The possible reason is described as follows.

For larger values of $\Gamma_{\text {th }}$, the effect of shock heating is stronger. This implies that the thermal energy of the HMNS is increased via the shock heating more efficiently, 

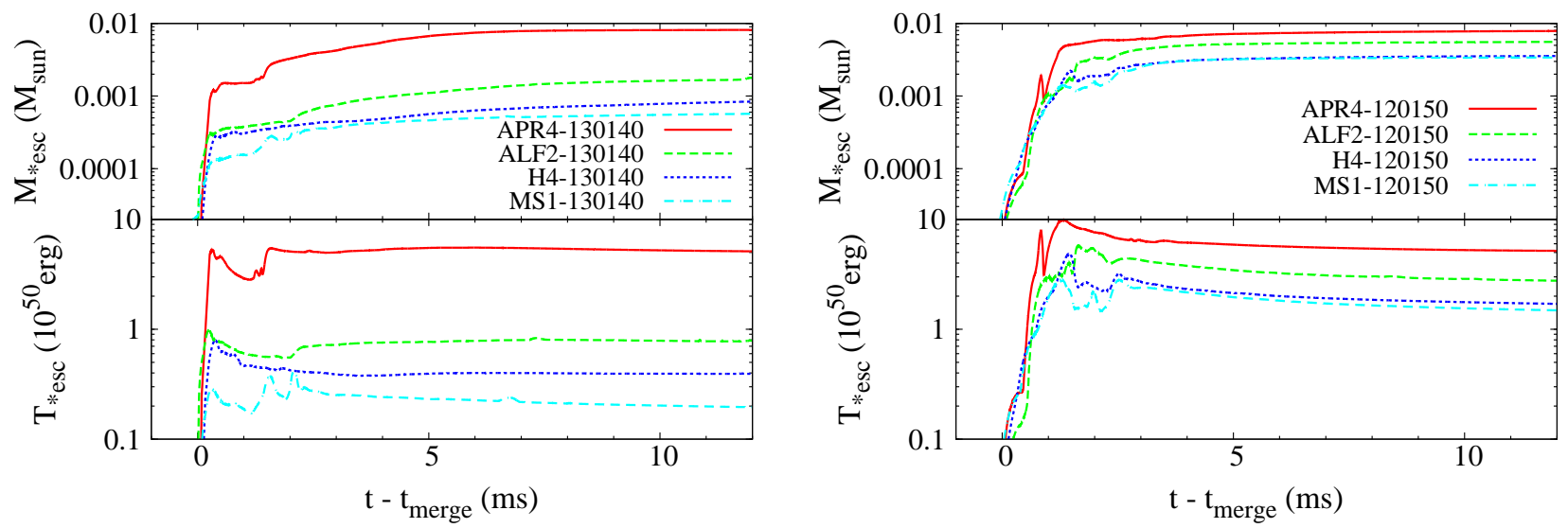

FIG. 12: $M_{* \text { esc }}$ and $T_{* \text { esc }}$ as functions of $t-t_{\text {merge }}$ (left) for models APR4-130140, ALF2-130140, H4-130140, MS1-130140, and (right) for models APR4-120150, ALF2-120150, H4-120150, MS1-120150.

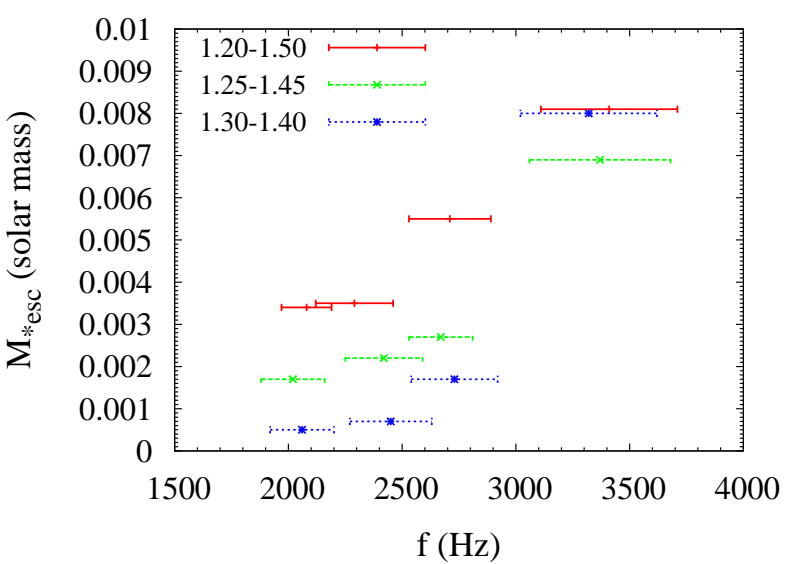

FIG. 13: $M_{* \text { esc }}$ as a function of the characteristic gravitational-wave frequency emitted by the HMNS. Here, the typical frequency is determined by the EOS; from the highest to the lowest, APR4, ALF2, H4, and MS1.

and thus, the material located outside the HMNS that will eventually escape from the system expands more efficiently at the merger and during the subsequent shock heating. This effect could result in increasing the ejected material.

On the other hand, the HMNS becomes less compact by more efficient shock heating for the larger value of $\Gamma_{\mathrm{th}}$, and hence, the amplitude of the quasiradial oscillation is smaller. This suggests that although the outward velocity of the material caused by the shock heating is initially larger for the larger values of $\Gamma_{\mathrm{th}}$, the subsequent gain of the kinetic energy via the shock heating could be smaller. The less compact HMNS could be also less favorable for exerting the torque to its surrounding material because the rotational velocity is slower. Therefore, the total rest mass and kinetic energy of the material ejected from the system depend on two competing nonlinear processes, as in a mechanism similar to that mentioned in Sec. IV A 3.
Figure 14 compares the evolution of $\bar{R}$ and $\bar{Z}$ for $\Gamma_{\mathrm{th}}=1.6,1.8$, and 2.0 for models APR4-135135 and APR4-120150. For APR4-135135, $\bar{R}$ is larger for the larger values of $\Gamma_{\text {th }}$ for $t-t_{\text {merge }} \gtrsim 1.5 \mathrm{~ms}$. This agrees with the prediction that the shock heating effect is stronger and the material expands in a wider region for the larger values of $\Gamma_{\mathrm{th}}$. For $\bar{Z}$, the similar result is found for $3 \lesssim t-t_{\text {merge }} \lesssim 5 \mathrm{~ms}$. However, for $t-t_{\text {merge }} \gtrsim 5 \mathrm{~ms}$, $d \bar{R} / d t$ and $d \bar{Z} / d t$ have a similar magnitude depending only weakly on the value of $\Gamma_{\mathrm{th}}$. This is due to the fact that the mass ejection is primarily driven by the torque exerted by the HMNS.

For APR4-120150, soon after the onset of the merger, $\bar{R}$ and $\bar{Z}$ are only slightly larger for the larger values of $\Gamma_{\text {th }}$. This is due to the fact that the mass ejection is primarily driven by the tidal effect caused by the mass asymmetry irrespective of the values of $\Gamma_{\mathrm{th}}$. However, for $t-t_{\text {merge }} \gtrsim 3 \mathrm{~ms}, d \bar{R} / d t$ and $d \bar{Z} / d t$ become smaller for the smaller values of $\Gamma_{\mathrm{th}}$. This is due to the fact that for the smaller value of $\Gamma_{\mathrm{th}}$, in particular, for $\Gamma_{\mathrm{th}}=1.6$, new materials with a smaller velocity are gradually ejected for the later time. This occurs due to the fact that for the lower value of $\Gamma_{\mathrm{th}}$, a longer-term mass ejection driven also by the torque exerted by the HMNS occurs. Namely, for both equal-mass and unequal-mass cases, a longterm mass ejection driven by the angular momentum transport from the HMNSs play an important role for $\Gamma_{\text {th }}=1.6$.

Figure 15 compares the evolution of the total rest mass and kinetic energy for $\Gamma_{\mathrm{th}}=1.6,1.8$, and 2.0 for models APR4-135135, APR4-120150, H4-135135, and H4120150. All the panels of Fig. 15 clearly show that for the early time, $t-t_{\text {merge }} \lesssim 1.5 \mathrm{~ms}$ for APR4-135135 and H4-135135, $55 \mathrm{~ms}$ for APR4-120150 and H4-120150, these two quantities are larger for the larger value of $\Gamma_{\mathrm{th}}$. Namely the stronger shock heating associated with the larger value of $\Gamma_{\text {th }}$ plays an important role. However, after the early time, the rest mass tends to be larger for the smaller value of $\Gamma_{\mathrm{th}}$. In particular, for $\Gamma_{\mathrm{th}}=1.6$, a rapid increase in the total rest mass is found. Thus, a 

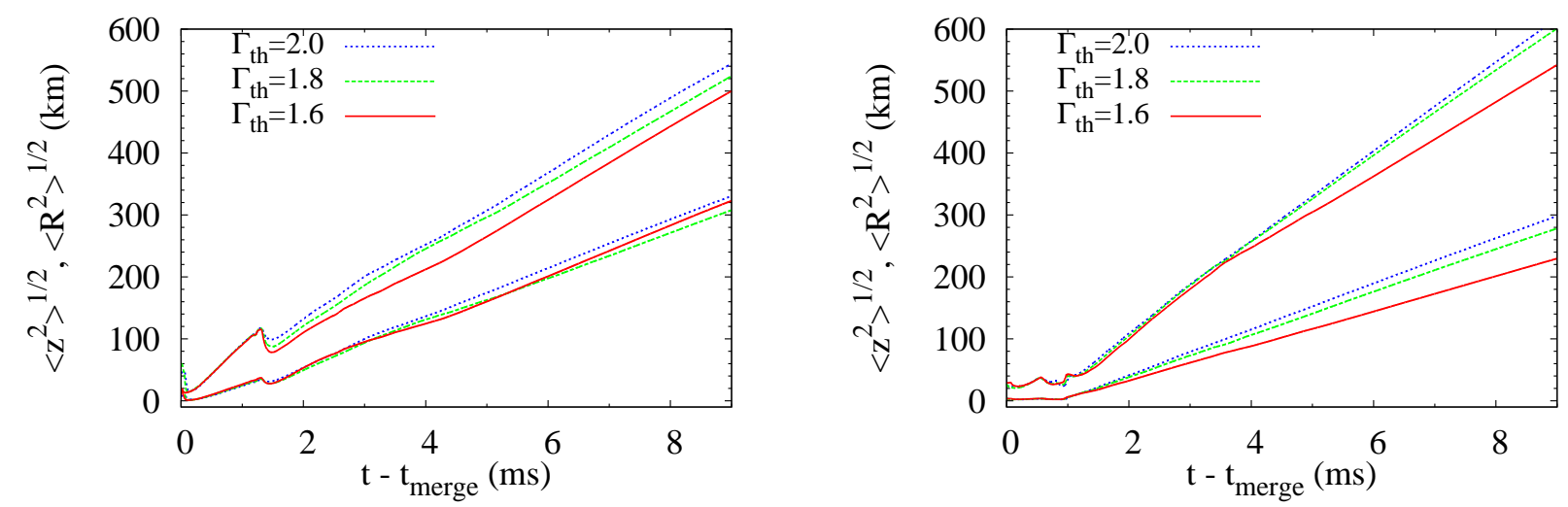

FIG. 14: $\bar{R}\left(=\left\langle R^{2}\right\rangle^{1 / 2}\right)$ (upper curves) and $\bar{Z}\left(=\left\langle z^{2}\right\rangle^{1 / 2}\right)$ (lower curves) as functions of time for models APR4-135135 (left) and APR4-120150 (right) with $\Gamma_{\text {th }}=2.0,1.8$, and 1.6.
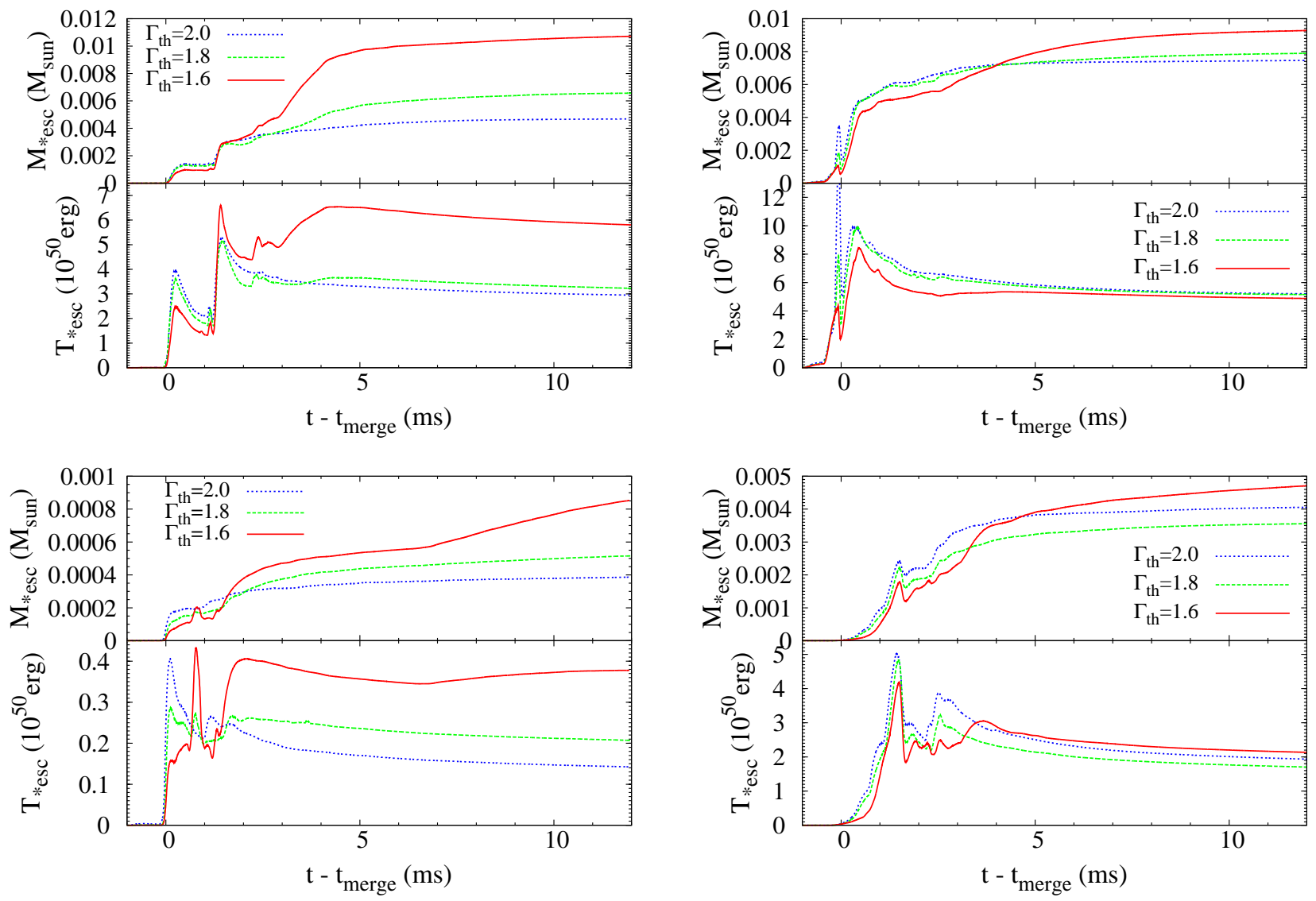

FIG. 15: $M_{* \text { esc }}$ and $T_{* \text { esc }}$ as functions of $t-t_{\text {merge }}$ (left) for models APR4-135135 (top left), APR4-120150 (top right), H4-135135 (bottom left), and H4-120150 (bottom right) with $\Gamma_{\text {th }}=2.0,1.8$, and 1.6.

longterm mass ejection process driven by the torque exerted by the HMNSs works for the smaller values of $\Gamma_{\text {th }}$ (i.e., for more compact HMNSs), and this mechanism is remarkable for $\Gamma_{\mathrm{th}}=1.6$.

For APR4-120150 and H4-120150, the rest mass of the ejected material is largest for $\Gamma_{\mathrm{th}}=1.6$. However, the kinetic energy depends weakly on the value of $\Gamma_{\text {th }}$. This im- plies that although more materials are ejected, the gained kinetic energy is not very large for $\Gamma_{\mathrm{th}}=1.6$, because the velocity of material ejected later by the tidal torque is not very large.

The dependence of the rest mass and kinetic energy of the ejected material on $\Gamma_{\mathrm{th}}$ is qualitatively similar for APR4 and H4. This indicates that the properties sum- 

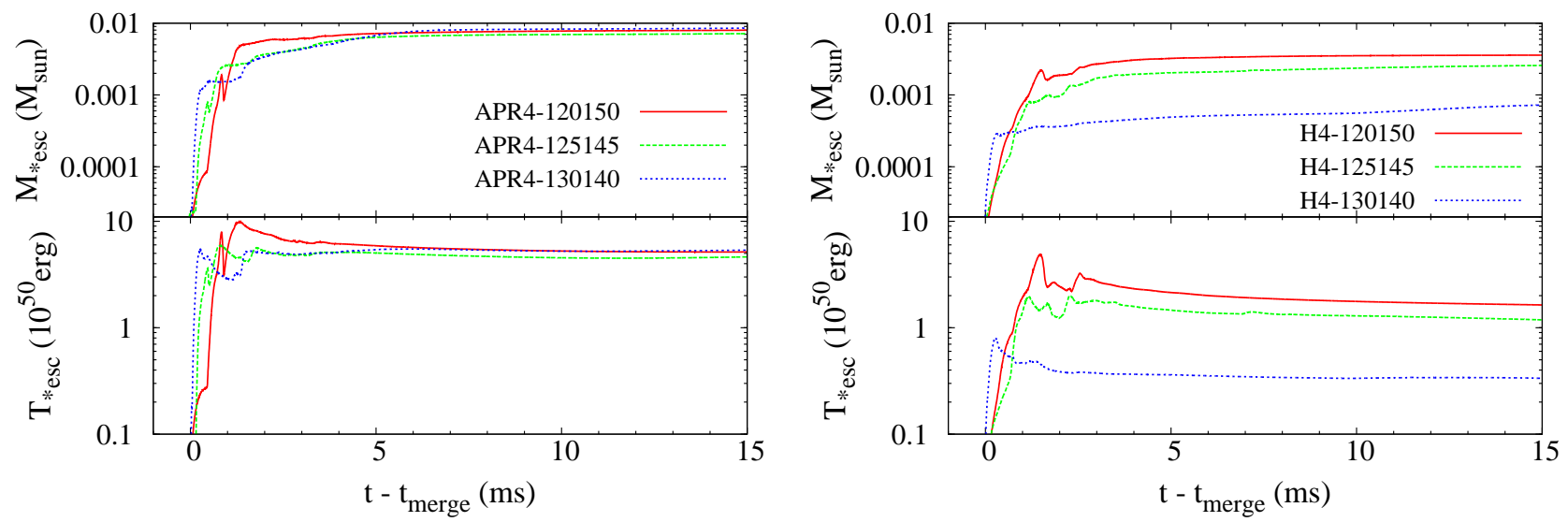

FIG. 16: $M_{* \text { esc }}$ and $T_{* \text { esc }}$ as functions of $t-t_{\text {merge }}$ (left) for models APR4-120150, APR4-125145, APR4-130140, and (right) for models H4-120150, H4-125145, H4-130140.

marized in this subsection would hold irrespective of the EOS.

\section{Dependence of the ejected material on the mass ratio and total mass}

The total rest mass and kinetic energy of the material ejected from the HMNSs depend also on the mass ratio and total mass of binary neutron stars. The degree of the dependence depends on the EOS. Figure 16 plots $M_{* \text { esc }}$ and $T_{* \text { esc }}$ as functions of $t-t_{\text {merge }}$ for APR4 and H4 with three mass ratios and with the total mass $2.7 M_{\odot}$. For the models with $\mathrm{H} 4$, the total rest mass and kinetic energy of the ejected material depend strongly on the mass ratio; e.g., the total rest mass and kinetic energy for $q=0.8$ are by a factor of $\sim 5$ and 7 larger than those for $q=0.929$ and $q=1$ with $m=2.7 M_{\odot}$. Essentially the same results are found for the models with ALF2 and MS1 with $q<1$ (see Table IV). By contrast, for the models with APR4, the total rest mass and kinetic energy depend weakly on the mass ratio for $m=2.7 M_{\odot}$, and they are always larger than those with ALF2, H4 and MS1 for $m=2.6-2.8 M_{\odot}$. These facts indicate that (i) for relatively stiff EOS such as ALF2, H4 and MS1, the asymmetry of binary neutron stars enhances the efficiency of the angular momentum transport via the tidal torque and increases the total amount of the ejected material, and (ii) for a relatively soft EOS, APR4, which yields a small-radius neutron star, the total amount of the ejected material is always large irrespective of the mass ratio for the canonical total mass $\sim 2.6-2.8 M_{\odot}$. This is probably because for APR4, the shock heating in the early evolution stage of the HMNSs, in which they quasiradially oscillate with significant amplitude, plays a primary role in the mass ejection irrespective of the mass ratio; indeed, a large mass ejection is observed in the first $1-2 \mathrm{~ms}$ after the onset of the merger.

The total rest mass and kinetic energy of the ejected material depend also on the total mass of the system, and the degree of the dependence depends also on the EOS: For APR4, these quantities are larger for more massive system irrespective of the mass ratio (see Table IV). This property is consistent with the fact that these quantities are larger for an EOS that yields compact neutron stars. Namely, for the larger mass, the system can be in general more compact for the binary neutron stars, and also the formed HMNS can be more compact, more rapidly rotate, and quasiradially oscillate with a larger amplitude. Thus, the mass ejection is enhanced through the angular momentum transport via the tidal torque and the shock heating.

For H4, the similar results are obtained except for model H4-140140. for which the rest mass and kinetic energy of the ejected material is smaller than those for models H4-135135. The possible reason is that for H4140140 (for which a black hole is formed $\sim 10 \mathrm{~ms}$ after the onset of the merger), the HMNS formed is compact (i.e., it can trap the material in its vicinity) and moreover, its shape (it is not a sharp ellipsoid) could be unsuitable for efficiently exerting the torque to the surrounding material.

For MS1 for which neutron stars and HMNSs are not very compact, the quantities of the ejected material do not change very systematically. As mentioned above, for $q<1$, the rest mass and kinetic energy increase with the decrease of $q$ in the same manner as that for other EOSs. However, for the equal-mass case $(q=1)$ with $m=2.6$ and $2.7 M_{\odot}$, the ejected rest mass and kinetic energy are quite large by contrast to that for $q=0.929$ and $m=2.7 M_{\odot}$. This indicates that for this system, the HMNS is not very compact and does not trap the material strongly, and hence, angular momentum transport due to the torque exerted by the HMNS and shock heating, which are not as efficient as those in softer EOSs, are still large enough to overcome the trapping effect. For $m=2.7 M_{\odot}$, in particular, the difference in the results of $q=1$ and $q=0.929$ is quite large. The possible reason 
is that (i) for the equal-mass case, the amplitude of the quasi-radial oscillation is by a factor of $\sim 2$ larger than that for the unequal-mass case, and thus, a larger amount of the materials are likely to gain the escape velocity; (ii) the shape of the HMNS is quite different between two models: For $q=1$, a sharp ellipsoid is formed, and it appears to play a substantial role for a coherent angular momentum transport from the HMNS to the material surrounding it. By contrast, for $q=0.929$, the shape is not a clear ellipsoid but a pear-shaped asymmetric object, and hence, the transport process does not appear to proceed efficiently.

For MS1-140140, the quantities of the ejected material is much smaller than those for MS1-135135 and as small as those for MS1-130140. The possible reason is that the HMNS formed for MS1-140140 is slightly more compact than that for MS1-135135, and the mass ejection is suppressed by the trapping effect. This suggests that for these mass ranges, a slight change in the compactness significantly affects the efficiency of the mass ejection.

\section{B. Properties of the merger and mass ejection: black hole formation case}

We briefly summarize the properties of the ejected material for the case that a black hole is promptly formed after the onset of the merger. In this study, the prompt formation of a black hole occurs only for APR4 with the total mass $2.9 M_{\odot}$.

For these models, the mass ejection primarily proceeds at the instance of the merger, i.e., during a short duration before the formation of a black hole. Because a black hole is promptly formed, a region shock-heated at the collision of two neutron stars is soon swallowed by the black hole, and thus, the shock heating does not play a primary role in the mass ejection. A significant mass ejection occurs for the case that the mass asymmetry is present, and the mass ejection is induced primarily by a tidal torque. In the presence of mass asymmetry, the less-massive neutron star is tidally elongated during the merger, and a fraction of the tidally elongated neutron-star material gains a sufficient torque from the merged object just before the formation of a black hole and gets the escape velocity. For models APR4-140150 and APR4-130160, this gain of the angular momentum is large enough to eject materials of rest mass $\sim 6 \times 10^{-4} M_{\odot}$ and $2 \times 10^{-3} M_{\odot}$, respectively (see Fig. 18). In these cases, disks are also formed, and their rest mass (for material bounded by the black hole) is $0.03 M_{\odot}$ and $0.002 M_{\odot}$, respectively. The values for the mass ejection depend only very weakly on the grid resolution with the fluctuation within $10-20 \%$ level (see Appendix A). The reason is that strong shocks do not play an important role in the mass ejection mechanism.

The average velocity of the ejected material for these cases is $\sim 0.3 c$ and larger than that in the case of the HMNS formation. The reason is that the mass ejection is caused primarily by the tidal interaction at the on- set of the merger, and for this case, the induced velocity is larger than that by subsequent longterm shock heating. Because the tidal interaction plays a primary role, the material is ejected primarily in the direction of the equatorial plane. The motion to the $z$ direction is also induced by shock heating that occurs when spiral arms surrounding the black hole collide each other. However, this is a secondary effect. Hence, for the case that a black hole is promptly formed from an asymmetric binary, the value of $\theta_{0}$ is $30-35^{\circ}$ which is smaller than those for the case of the HMNS formation for which $\theta_{0}=40-50^{\circ}$.

For the equal-mass binary, the total rest mass of the ejected material is quite small $\sim 10^{-4} M_{\odot}$ (see Fig. 18), because of the absence of the asymmetry and of the lack of the time during which the material located in the outer region gains the torque from the merged object (note that most of the fluid elements of binary neutron stars just before the onset of the merger do not have the specific angular momentum large enough to escape from the black hole formed [21]). In this case, the disk mass surrounding the black hole is also quite small, $\sim 10^{-4} M_{\odot}$. This is consistent with our previous finding [25].

Figure 19 plots the gravitational waveforms for APR4130160 and APR4-140150. For these models (also for APR4-145145), the gravitational waveform is characterized by the inspiral waveform and subsequent ringdown waveform. The frequency of gravitational waves monotonically increases and eventually reaches the value of the fundamental quasinormal mode of the formed black hole. For all three cases, the frequency of gravitational waves associated with the quasinormal mode is $6.55 \pm 0.05 \mathrm{kHz}$ (the mass and spin of the formed black holes are $2.8-$ $2.83 M_{\odot}$ and $0.77-0.78$, respectively), which agrees with the frequency of the quasinormal mode analytically derived 52. Because HMNS is not formed, no feature for the quasiperiodic oscillation associated with the HMNS formation is found.

\section{SUMMARY AND DISCUSSION}

\section{A. Summary}

We reported our latest numerical-relativity studies for the material ejected in the merger of binary neutron stars. We explored the properties of the ejected material for a variety of EOSs, total masses, and mass ratios of binary neutron stars, and found the following facts. First, we summarize the results for the case that a HMNS is formed:

- For the canonical total mass of the binary neutron stars $2.6-2.8 M_{\odot}$, the total rest mass and kinetic energy of the ejected material are approximately in the range $10^{-4}-10^{-2} M_{\odot}$ and $10^{49}-10^{51}$ ergs, respectively, depending on the EOS, total mass, and mass ratio of binary neutron stars.

- The total rest mass and kinetic energy of the 


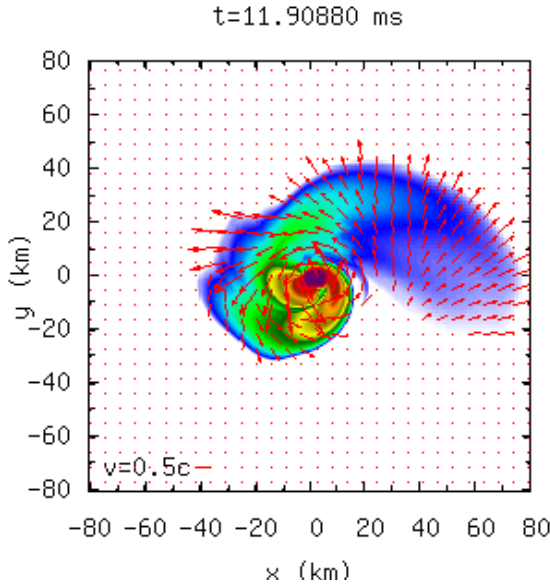

$\mathrm{t}=13.74092 \mathrm{~ms}$
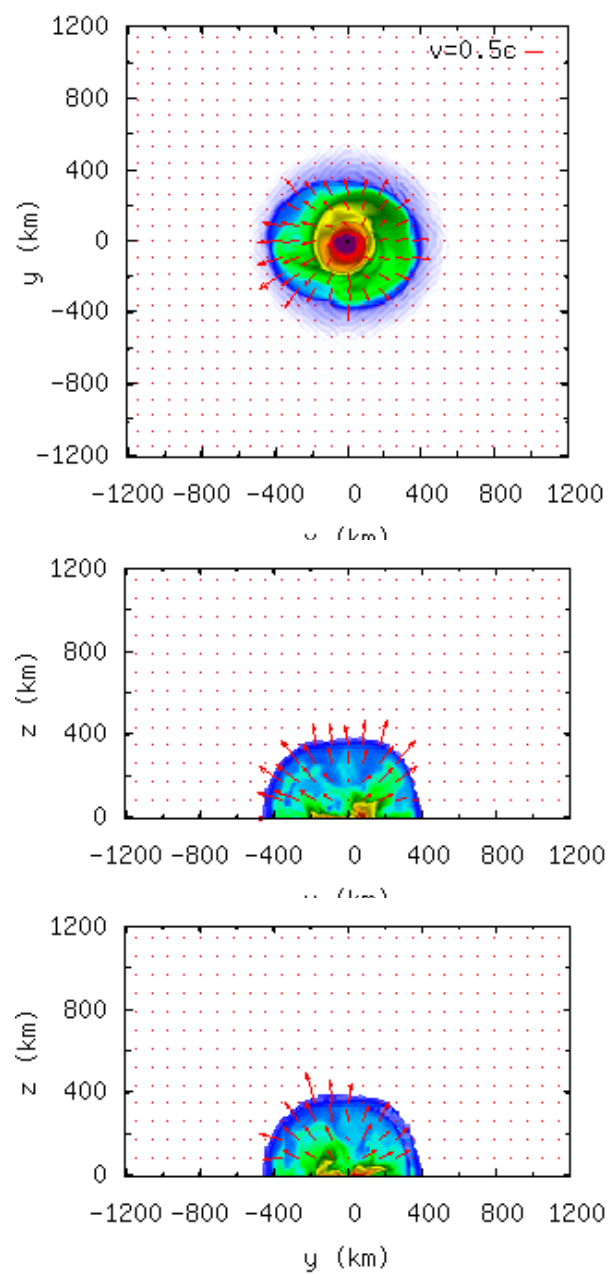

$\mathrm{t}=12.82486 \mathrm{~ms}$

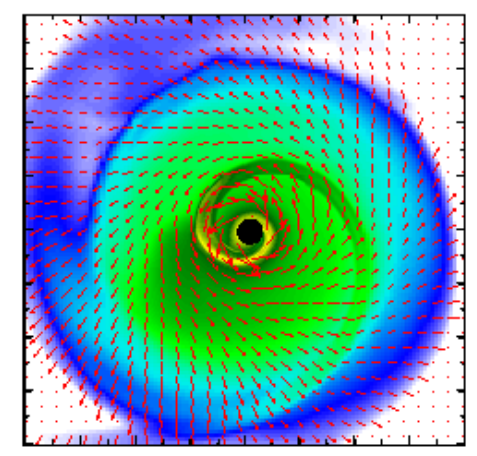

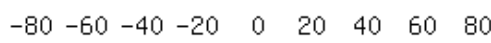

$\times(\mathrm{km})$

$\mathrm{t}=15.34403 \mathrm{~ms}$
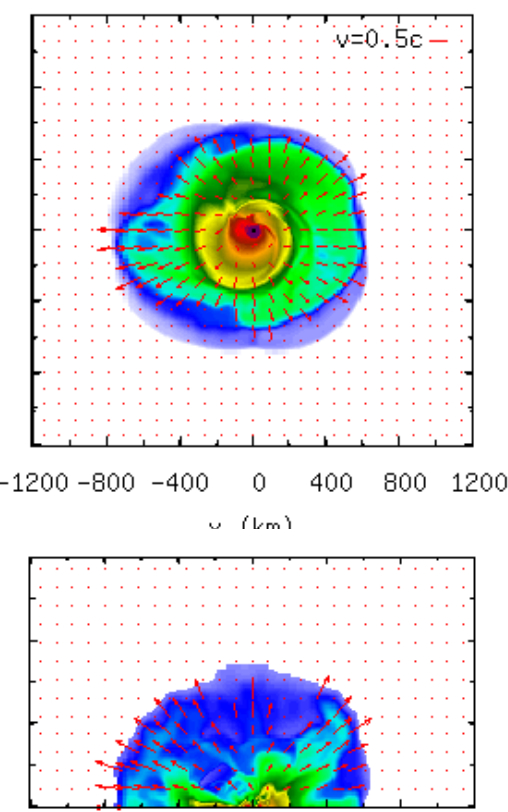

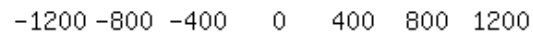

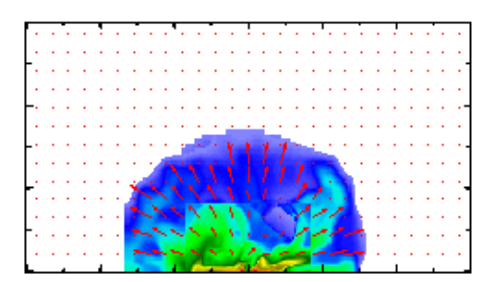

$\begin{array}{llllll}-1200-800 & -400 \quad 0 & 400 & 800 & 1200\end{array}$

y $(\mathrm{km})$

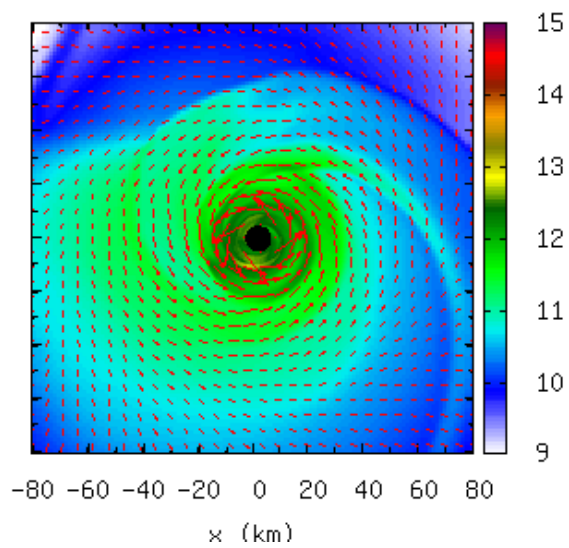

$\mathrm{t}=16.94714 \mathrm{~ms}$
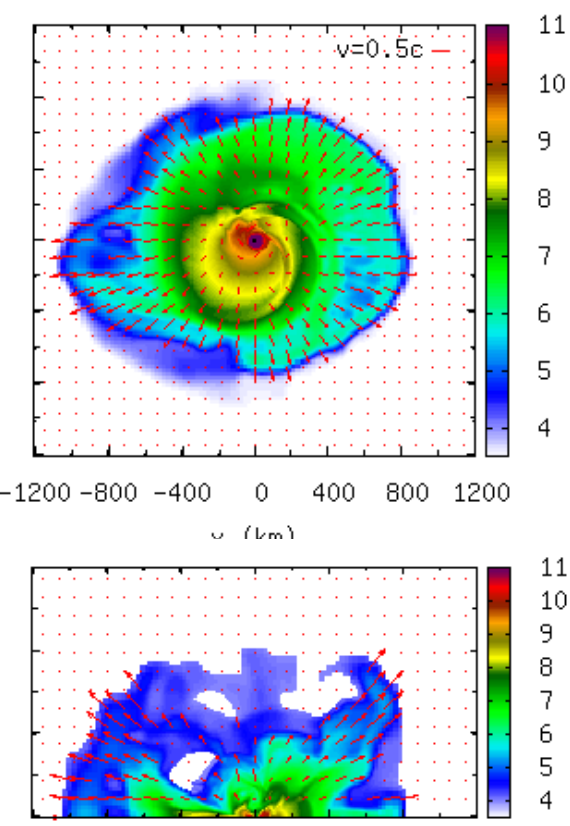

$\begin{array}{llllll}-1200 & -800 & -400 \quad 0 & 400 & 800 & 1200\end{array}$

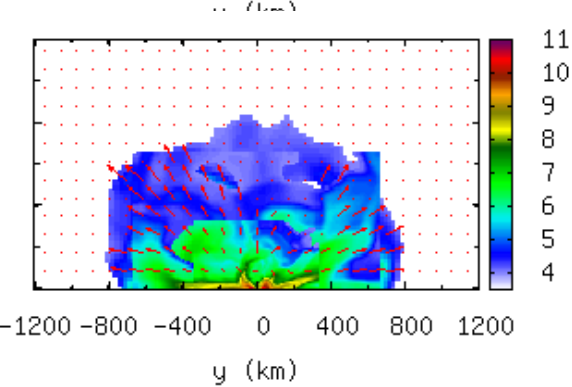

FIG. 17: The same as Fig. 3 but for high-mass and unequal-mass model APR4-130160. The filled black circles in the middle and right panels of the top row denote black holes. 


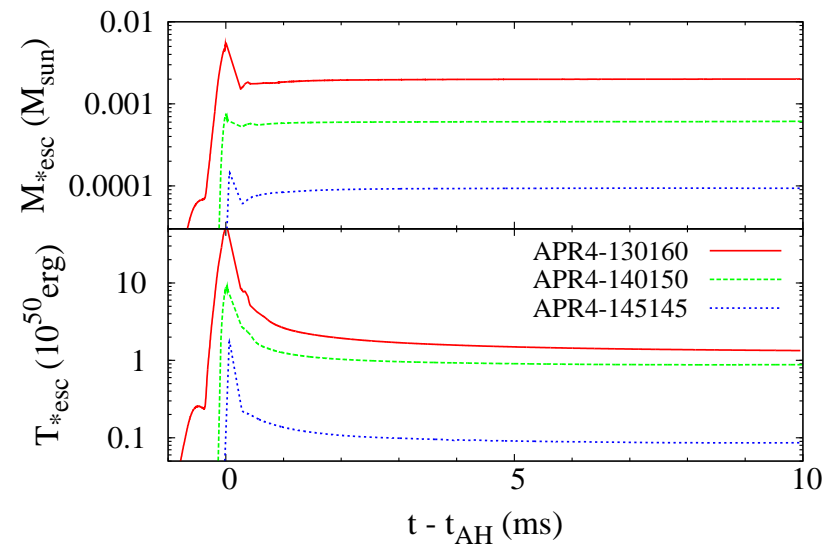

FIG. 18: $M_{* \text { esc }}$ and $T_{* \text { esc }}$ as functions of $t-t_{\mathrm{AH}}$ for models APR4-130160, APR4-140150, APR4-145145. Here, $t_{\mathrm{AH}}$ denotes the time at which an apparent horizon is formed.

ejected material depend strongly on the EOS. They are in general larger for binaries composed of an EOS that yields compact (small-radius) neutron stars (e.g., APR4). They also depend on the efficiency of shock heating (i.e., depend on $\Gamma_{\text {th }}$ ): For many cases, smaller values of $\Gamma_{\text {th }}$ results in a larger ejected mass and kinetic energy.

- The total rest mass and kinetic energy could depend also strongly on the mass ratio of binary neutron stars. The dependence on the mass ratio is in particular strong when the radius of neutron stars is relatively large (i.e., for stiff EOSs such as H4 and MS1). For many models in such EOSs, the ejected rest mass and kinetic energy are larger for smaller mass ratios (for more asymmetric binary neutron stars). By contrast, when the EOS is relatively soft (i.e., for APR4), the dependence of these quantities on the mass ratio is weak.

- The total rest mass and kinetic energy depend also on the total mass of binary neutron stars. For many models in the present study, these quantities are larger for the larger total mass irrespective of the EOS. However, there are some exceptions for a class of EOS and for a massive system.

- The total rest mass ejected is in the wide range between $\sim 5 \times 10^{-4}(\mathrm{H} 4)$ and $\sim 7 \times 10^{-3} M_{\odot}$ (APR4) for equal-mass binaries with the total mass $m=2.7 M_{\odot}$. For the unequal-mass case with $q \approx 0.8$, it is in a rather narrow range between $\sim 3 \times 10^{-3}$ (MS1 and $\left.\mathrm{H} 4\right)$ and $\sim 8 \times 10^{-3} M_{\odot}$ (APR4) for $m=2.7 M_{\odot}$. This indicates that the ejection of the material is induced by two different processes, the torque exerted the HMNS and the shock heating.

- The total kinetic energy is also in the wide range between $\sim 10^{49} \mathrm{ergs}\left(\mathrm{MS} 1\right.$ and H4) and $\sim 10^{51} \mathrm{ergs}$
(APR4). The typical maximum velocity of the ejected material is $0.5-0.8 c$, and the average velocity is $0.15-0.25 c$. For EOSs that yield a compact neutron star, the velocity of escaping material is larger.

In our present study, a black hole is promptly formed for three models; APR4-145145, APR4-140150, and APR4-130160. For the case of the prompt black-hole formation, a significant mass ejection occurs only for the asymmetric binaries. For APR4-145145, the rest mass of the ejected material is $\sim 10^{-4} M_{\odot}$. By contrast, it is rather large for APR4-140150 and APR4-130160 as $6 \times 10^{-4} M_{\odot}$ and $2 \times 10^{-3} M_{\odot}$. Thus, a massive and asymmetric binary can eject a large amount of the material even if a black hole is promptly formed (even in the absence of a HMNS). For this case, the average velocity of the ejected material is larger than that for the case of the HMNS formation, and thus, a unique feature may be seen in the observed electromagnetic signal (see next section). However, we should note that the prompt black-hole formation will occur only for a large total mass, because the latest discovery of a high-mass neutron star PSR J16142230 with mass $1.97 \pm 0.04 M_{\odot}[29$ indicates that the EOS should be rather stiff and with such stiff EOSs, a HMNS is the canonical outcome for the canonical-mass binary with $m=2.6-2.8 M_{\odot}$.

The distribution of the matter around the remnant object depends strongly on the merger process. For the case that a HMNS is formed, a dense (physical) atmosphere (including the ejected material) is formed around it (see Figs. 3- 57. The atmosphere is distributed in a weakly anisotropic manner, and thus, the matter is present even in the spin axis of the HMNS. Such dense atmosphere will be present even after the HMNS collapses to a black hole. HMNS or black hole subsequently formed will emit a huge amount of neutrinos (e.g., [27]), and may drive a jet from the central region through the fireball production via neutrino-antineutrino pair annihilation. To drive a SGRB for this case, however, the jet has to penetrate the dense atmosphere and ejected material. Whether it is possible or not is a question to be answered by the future research. By contrast, for the case of the black hole formation, the atmosphere is not very dense around the spin axis of the black hole (see Fig. 17). Thus, for this case, a SGRB would be driven, if an energetic jet is launched as studied, e.g., in [53].

\section{B. Discussion}

We here briefly argue possible electromagnetic signals emitted by the material ejected from the merger of binary neutron stars, referring to the numerical results in the present work. As already mentioned, a recent discovery of a high-mass neutron star PSR J1614-2230 suggests that the maximum mass of spherical neutron stars should be larger than $1.97 \pm 0.04 M_{\odot}[29$. This indicates that a long-lived HMNS would be a canonical outcome of the 

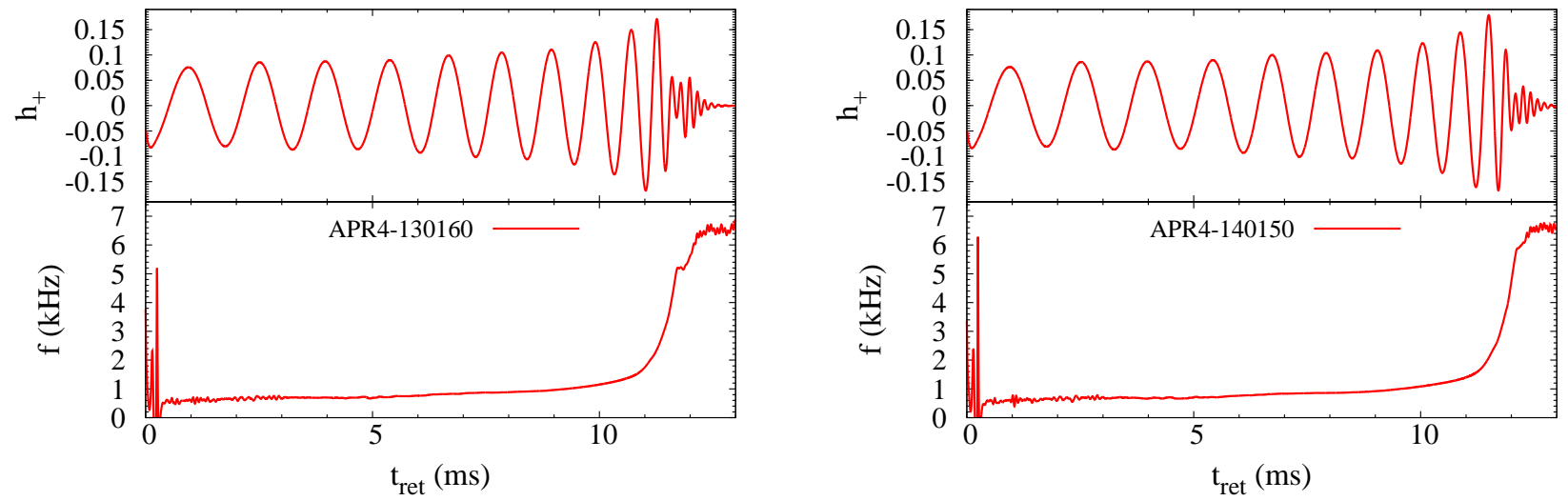

FIG. 19: The same as Fig. 9 but for models APR4-130160 (left) and APR4-140150 (right).

merger of binary neutron stars, if the binaries were composed of neutron stars of canonical mass of $1.3-1.4 M_{\odot}$ with the total mass $\sim 2.6-2.8 M_{\odot}[26$. The present numerical results indicate that from the long-lived HMNS, a fraction of the material could be ejected with large kinetic energy.

References [1,3, 18, discuss the signals by the radioactive decay of $r$-process nuclei, which would be produced from the neutron-rich material in the outflow [12 17, and subsequently decay and emit a signal that may be observable by current and future-planned optical telescopes such as PTF [54, Pan-STARRs 55, and LSST 56. In this scenario, the typical duration of the peak luminosity is of order a day or less as [1]

$$
t_{\text {peak }} \approx 0.1 \mathrm{~d}\left(\frac{\beta_{0}}{0.2}\right)^{-1 / 2}\left(\frac{M_{* \mathrm{esc}}}{10^{-3}}\right)^{1 / 2}
$$

and the associated peak luminosity is

$$
\begin{aligned}
L_{\text {peak }} \approx 7 \times 10^{41} & \text { ergs } / \mathrm{s}\left(\frac{f_{\text {eff }}}{3 \times 10^{-6}}\right) \\
& \times\left(\frac{\beta_{0}}{0.2}\right)^{1 / 2}\left(\frac{M_{* \mathrm{esc}}}{10^{-3} M_{\odot}}\right)^{1 / 2}
\end{aligned}
$$

where $f_{\text {eff }}$ denotes the conversion rate of the energy per rest-mass energy in the ejected material through the radioactive decay process, which is $\sim 3 \times 10^{-6}$ according to the results of [3]. $\quad \beta_{0} c$ is the typical velocity of the ejected material. The result of 3 suggests that if the total ejected mass is $\gtrsim 10^{-3} M_{\odot}$, the signal will be detected by large optical surveys such as LSST for a typical distance to sources $\sim 100 \mathrm{Mpc}$. Our numerical results indicate that $\beta_{0}=0.15-0.25$, and the total ejected mass is $\sim 10^{-3}-10^{-2} M_{\odot}$ for binaries composed of neutron stars with a small radius $\sim 11-12.5 \mathrm{~km}$, and $\sim 0.3 \times 10^{-3}$ $5 \times 10^{-3} M_{\odot}$ for binaries composed of neutron stars with a larger radius $\sim 13.5-14.5 \mathrm{~km}$ for plausible values of $\Gamma_{\mathrm{th}}=1.6-2.0$. Thus, if the EOS is a rather "soft" one that yields a small-radius neutron star, an observable optical signal due to the radioactive decay can be expected with a duration of several hours. Taking into account the short duration of the signal, rapid follow-up searches and an efficient coverage for the error circle of the direction of the gravitational-wave events are required. If the EOS is a "stiff" one that yields a large-radius neutron star, the strength of the signal will be weaker and the duration shorter, although it would be still possible to detect the signal in particular for the merger of unequal-mass (sufficiently asymmetric) neutron stars.

There is also another possible channel for the electromagnetic emission. According to recent studies [4, 18, 20], the ejected material, which is in the free expansion, will sweep up the interstellar matter and form blast waves. During this process turning on, the shocked material could generate magnetic fields and accelerate particles that emit synchrotron radiation, for a hypothetical amplification of the electromagnetic field and a hypothetical electron injection. The emission will peak when the total swept-up mass approaches the ejected mass, because the blast waves are decelerated and transit to the phase in which the motion of the material is described by the (non-relativistic) Sedov-Taylor's self-similar solution. The predicted duration for the synchrotron radiation depends on the total energy $E_{0}$ and speed of the ejected material $\beta_{0} c$ as well as the number density of the interstellar matter $n_{0}$. The duration to reach the peak luminosity is estimated in [4] as

$\tau_{\text {radio }} \sim 4 \operatorname{yrs}\left(\frac{E_{0}}{10^{50} \mathrm{ergs}}\right)^{1 / 3}\left(\frac{n_{0}}{1 \mathrm{~cm}^{-3}}\right)^{-1 / 3}\left(\frac{\beta_{0}}{0.2}\right)^{-5 / 3}$.

By the synchrotron radiation, a radio signal could be emitted as in the late phase of supernovae and the afterglow of gamma-ray bursts [4. Our numerical results indicate that the typical velocity of the ejecta is $\beta_{0}=0.15$ - 0.25 irrespective of the EOS and masses of neutron stars in binaries. However, $E_{0}$ is in a wide range between $\sim 10^{49}$ ergs and $10^{51}$ ergs, depending strongly on the EOS, mass ratio, and total mass of the binaries, and its value is highly uncertain. Thus the predicted value 
of $\tau_{\text {radio }}$ is in a wide range $\sim 1-10$ yrs, even for an optimistic value of $n_{0}=1 \mathrm{~cm}^{-3}$. For smaller values of $n_{0}$ which is likely when the merger occurs outside the galactic plane, the value of $\tau_{\text {radio }}$ is much longer.

For the typical value of the ejecta velocity $\beta_{0} \sim 0.2$, the peak flux for the observed frequency is obtained at the deceleration time described in Eq. 31. Specifically, the peak flux may be obtained at the self-absorption frequency, $\sim 1-2$ hundreds $\mathrm{MHz}$, and the typical synchrotron frequency is sub-MHz. The peak flux for a given observed radio-band frequency $\nu_{\mathrm{obs}}$ is

$$
\begin{aligned}
F_{\nu} \approx & 90 \mu \mathrm{Jy}\left(\frac{E_{0}}{10^{50} \mathrm{ergs}}\right)\left(\frac{n_{0}}{1 \mathrm{~cm}^{-3}}\right)^{0.9}\left(\frac{\beta_{0}}{0.2}\right)^{2.8} \\
& \times\left(\frac{D}{200 \mathrm{Mpc}}\right)^{-2}\left(\frac{\nu_{\mathrm{obs}}}{1.4 \mathrm{GHz}}\right)^{-0.75}
\end{aligned}
$$

where we assumed the power-law distribution of the electron's Lorentz factor with the power 2.5. Equation (32) is applicable as long as the observed frequency is higher than the typical synchrotron and self-absorption frequency at the deceleration time, $\tau_{\text {radio. Equation } 32}$ indicates that for a hypothetical event at a distance of $200 \mathrm{Mpc}, E_{0} \sim 10^{50}$ ergs with $n_{0}=1 \mathrm{~cm}^{-3}$ is strong enough to be observed by future-planned radio instruments (such as EVLA [57], ASKAP [58], MeerKAT [59], and Apertif for which the root-mean square value of the background noise for one hour observation is smaller than $50 \mu \mathrm{Jy}$ as shown in 4]). Therefore, the mass-ejection mechanism could supply a large amount of the kinetic energy which generates an observable strong radio signal, if the EOS is rather soft (i.e., the neutron-star radius is fairly small) or the binary is significantly asymmetric.

In this scenario, the duration to reach the peak luminosity and the strength of the radio signal depend strongly on the value of $n_{0}$. In nature, the value of $n_{0}$ will depend strongly on the site where the merger of binary neutron stars happens. If it is in a galactic disk, $n_{0}$ would be typically $\sim 1 \mathrm{~cm}^{-3}$, whereas if it is outside a galaxy, the value is much smaller as $\sim 10^{-3} \mathrm{~cm}^{-3}$. Equation (32) shows that for a smaller value of $n_{0} \ll 1 \mathrm{~cm}^{-3}$, $F_{\nu} \lesssim 1 \mu \mathrm{Jy}$ even for $E_{0}=10^{51}$ ergs. Our numerical simulation shows that the maximum value of $E_{0}$ is at most $10^{51}$ ergs. Therefore, for the low value of $n_{0} \sim 10^{-3} \mathrm{~cm}^{-3}$, this type of electromagnetic signals may not be observable as a counterpart of the gravitationalwave signal [18.

We here note the following point. We used the total kinetic energy and average velocity as $E_{0}$ and $\beta_{0}$, when estimating the radio flux estimated in Eq. (32). However, as we found in this paper, the ejected material has a wide range of the velocity, and the amount of the kinetic energy which the material of a given value of $\beta_{0}$ has depends on the value of $\beta_{0}$. Thus, each material has a different deceleration time and flux $F_{\nu}$, and therefore, the light curve will have a complicated structure depending on the distribution of the differential mass as a function of the ejecta velocity (see also [19]). In a subsequent pa- per, we plan to study the luminosity curve in more detail following 20].

As summarized in Sec. $\mathrm{VA}$ the properties of the ejected material depend strongly on the EOS, mass ratio, and total mass of the binary. This suggests that the observed electromagnetic signal depends on them. The observation of gravitational waves in the inspiral phase of binaries, which will be observed in the advanced gravitational-wave detectors [5], will carry the information of the mass ratio and total mass. The observation of gravitational waves from the final inspiral phase and HMNS could constrain the EOS of neutron stars. Together with these information by the gravitational-wave observation, the observation of the electromagnetic signals will be used for clarifying the dynamics of the binary merger and ejected material. In addition, clarifying the spectroscopic properties of electromagnetic emission associated with the decay of $r$-process nuclei may be interesting. If there is a characteristic emission/absorption feature in this emission, it will be helpful to determine the cosmological redshift of the source event through a spectroscopic observation. The redshift determined will be subsequently used for determining the distance to the source (and thus, Hubble constant) and the physical mass of binary systems through the analysis of observed inspiral gravitational waves 60. However, for the clarification, detailed theoretical studies for the ejected material and electromagnetic radiation are necessary for a variety of the EOS, mass ratio, and total mass. These are new and interesting tasks for the community of numerical relativity.

\section{Acknowledgments}

We are grateful to T. Piran for suggesting to explore the mass ejection in detail, and K. Ioka, E. Nakar, and Y. Suwa for helpful discussions. This work was supported by Grant-in-Aid for Scientific Research (21340051, 21684014, 23740160, 24244028, 24740163), by Grant-in-Aid for Scientific Research on Innovative Area (20105004), and HPCI Strategic Program of Japanese MEXT. The work of Hotokezaka is supported by the Grant-in-Aid of JSPS.

\section{Appendix A: Convergence}

The rest mass and kinetic energy of the ejected material with different grid resolutions for selected models are listed in Table $\mathrm{V}$. This shows that for the unequalmass models, a convergence is well achieved (even for $m_{1}=1.3 M_{\odot}$ and $\left.m_{2}=1.4 M_{\odot}\right)$, whereas for the equalmass models, the convergence is poor. In this case, the results with $N>60$ could be modified by a factor of $\sim 2$ from the results with $N=60$. As mentioned in Sec. IV A, the convergence is poor for the case that a strong shock is formed at the merger and it plays a primary role in the 
mass ejection. The possible reason for this is that (i) the shocks are always computed by the first-order accuracy, and hence, the accuracy is low and (ii) the ejected mass is a tiny part of the entire system, and hence, a random error for the entire system computed with a low accuracy significantly (and randomly) affects a tiny amount of the ejected material. By contrast, when the tidal torque and hydrodynamical torque exerted by the HMNS play an important role in the mass ejection, the convergence is good.

The averaged frequency of gravitational waves emitted by HMNSs is also listed for three grid resolutions. This shows that the frequency is obtained within the error of $\lesssim$ $0.1 \mathrm{kHz}$, which is smaller than the physical dispersion of the frequency associated with the quasiradial oscillation of the HMNSs and the secular change of the density and velocity profiles of the HMNSs caused by the angular momentum transport.
[1] L. Li and B. Paczyński, Astrophys. J. 507, L59 (1998).

[2] S. R. Kulkarni, astro-ph:0510256.

[3] B. D. Metzger, G. Martinez-Pinedo, S. Darbha, E. Quataert, A. Arcones, D. Kasen, T. Thomas, P. Nugent, I. V. Panov, and N. T. Zinner, Mon. Not. Roy. Soc. 406, 2650 (2010).

[4] E. Nakar and T. Piran, Nature 478, 82 (2011); arXiv:1102.1020

[5] J. Abadie, et al., Nucl. Instrum. Meth. A624, 223 (2010): T. Accadia, et al., Class. Quantum Grav. 28, 025005 (2011): K. Kuroda, et al., Class. Quantum Grav. 27, 084004 (2010).

[6] V. Kalogera et al., Phys. Rep. 442, 75 (2007).

[7] J. Abadie et al., Class. Quantum Grav. 27, 173001 (2010).

[8] R. Narayan, B. Paczynski, and T. Piran, Astrophys. J. Lett. 395, L83 (1992): T. Piran, Rev. Mod. Phys. 76, 1143 (2005): E. Nakar, Phys. Rep. 442, 166 (2007).

[9] W. Fong et al., Astrophys. J. 756, 189 (2012).

[10] S. L. Liebling, L. Lehner, D. Neilsen and C. Palenzuela, Phys. Rev. D 81, 124023 (2010)

[11] L. Rezzolla, B. Giacomazzo, L. Baiotti, J. Granot, C. Kouveliotou and M. A. Aloy, Astrophys. J. 732, L6 (2011).

[12] L. F. Roberts, D. Kasen, W. H. Lee, E. Ramirez-Ruiz, Astrophys. J. 736 L21 (2011)

[13] S. Goriely, A. Bauswein, and H.-T. Janka, Astrophys. J. Lett. 738, L32 (2011).

[14] S. Rosswog, M. Liebendorfer, F. -K. Thielemann, M. B. Davies, W. Benz, and T. Piran, A\&A, 341, 499 (1999)

[15] C. Freiburghaus, S. Rosswog, and F. -K. Thielemann, Astrophys. J. 525, L121 (1999)

[16] S. Rosswog, M. B. Davies, F. -K. Thielemann, and T. Piran, A\&A, 360, 171 (2000)

[17] S. Rosswog, arXiv:1210.6549

[18] B. D. Metzger and E. Berger, Astrophys. J. 746, 48 (2012).

[19] K. Kyutoku, K. Ioka, and M. Shibata, arXiv:1209.5747

[20] T. Piran, E. Nakar, and S. Rosswog, arXiv:1204.6242.

[21] M. Shibata and K. Uryū, Phys. Rev. D 61, 064001 (2000).

[22] M. D. Duez, Class. Quantum Grav. 27, 114002 (2010).

[23] J. A. Faber and F. A. Radio, Living Rev. Relativ. 15, 8 (2012).

[24] J. A. Font, Living Rev. Relativ. 11, 7 (2008).

[25] M. Shibata, K. Taniguchi, and K. Uryū, Phys. Rev. D 71, 084021 (2005); M. Shibata and K. Taniguchi, Phys. Rev. D 73, 064027 (2006); K. Kiuchi, Y.I. Sekiguchi, M. Shibata, and K. Taniguchi, Phys. Rev. D 80, 064037 (2009).

[26] K. Hotokezaka, K. Kyutoku, H. Okawa, M. Shibata, and
K. Kiuchi, Phys. Rev. D 83, 124008 (2011).

[27] Y. Sekiguchi, K. Kiuchi, K. Kyutoku, and M. Shibata, Phys. Rev. Lett. 107, 051102 (2011); 107, 211101 (2011).

[28] J. M. Lattimer and M. Prakash, Science 304, 536 (2004); Phys. Rep. 442, 109 (2007).

[29] P. Demorest, T. Pennucci, S. Ransom, M. Roberts, and J. Hessels, Nature 467, 1081 (2010).

[30] J. S. Read, B. D. Lackey, B. J. Owen, and J. L. Friedman, Phys. Rev. D 79, 124032 (2009).

[31] J. S. Read, C. Markakis, M. Shibata, K. Uryū, J. D. E. Creighton, and J. L. Friedman, Phys. Rev. D 79, 124033 (2009).

[32] K. Kyutoku, M. Shibata, and K. Taniguchi, Phys. Rev. D 82, 044049 (2010)

[33] K. Kyutoku, H. Okawa, M. Shibata, and K. Taniguchi, Phys. Rev. D 84, 064018 (2011).

[34] J. M. Lattimer and M. Prakash, Astrophys. J. 550, 426 (2001).

[35] A. Akmal, V. R. Pandharipande, and D. G. Ravenhall, Phys. Rev. D 58, 1804 (1998).

[36] H. Müller and B. D. Serot, Nucl. Phys. A 606, 508 (1996).

[37] N. K. Glendenning and S. A. Moszkowski, Phys. Rev. Lett. 67, 2414 (1991); B. D. Lackey, M. Nayyar, B. J. Owen, Phys. Rev. D 73, 024021 (2006).

[38] M. Alford, M. Brady, M. Paris, and S. Reddy, Astrophys. J 629, 969 (2005).

[39] K. Taniguchi and M. Shibata, Astrophys. J. Supple. 188, 187 (2010).

[40] I. H. Stairs, Science 304, 547 (2004); D. R. Lorimer, Living Rev. Relativ., 11, 8 (2008).; J. M. Lattimer and M. Prakash, arXiv:1012.3208

[41] http://www.lorene.obspm.fr/

[42] T. Yamamoto, M. Shibata, and K. Taniguchi, Phys. Rev. D 78, 064054 (2008).

[43] L. Baiotti, M. Shibata, and T. Yamamoto Phys. Rev. D 82, 064015 (2010).

[44] M. Shibata and T. Nakamura, Phys. Rev. D 52, 5428 (1995); T. W. Baumgarte and S. L. Shapiro, Phys. Rev. D 59, 024007 (1998); M. Campanelli, C. O. Lousto, P. Marronetti, and Y. Zlochower, Phys. Rev. Lett. 96, 111101 (2006); J. G. Baker, J. Centrella, D.-I. Choi, M. Koppitz, and J. van Meter, Phys. Rev. Lett. 96, 111102 (2006).

[45] B. Brügmann, J. A. Gonzalez, M. Hannam, S. Husa, U. Sperhake, and W. Tichy, Phys. Rev. D 77, 024027 (2008).

[46] A. Kurganov and E. Tadmor, J. Comp. Phys. 160, 241 (2000).

[47] A. Bauswein, H.-Th. Janka, and R. Oechslin, Phys. Rev. D 82, 084043 (2010).

[48] C. Reisswig and D. Pollney, Class. Quantum Grav. 28, 195015 (2011) 
TABLE V: Convergence for the rest mass and kinetic energy of the ejected material and average gravitational-wave frequency for selected models. For each column, $\left(M_{* \mathrm{esc}}, E_{* \mathrm{esc}}, f_{\mathrm{ave}, 5 \mathrm{~ms}}, f_{\mathrm{ave}, 10 \mathrm{~ms}}\right)$ in units of $10^{-3} M_{\odot} 10^{50}$ ergs, and $\mathrm{kHz}$ are listed. In this table, the values for the ejected material are shown in 2 significant digits.

\begin{tabular}{c|cccc}
\hline Model & $N=40$ & $N=48$ & $N=50$ & $N=60$ \\
\hline \hline APR4-130160 & $(2.3,1.8,-,-)$ & $(2.5,2.0,-,-)$ & - & $(2.0,1.5,-,--)$ \\
APR4-120150 & $(8.0,5.4,3.29,3.30)$ & $(8.4,5.7,3.30,3.28)$ & - & $(8.0,5.2,3.41,3.35)$ \\
APR4-130140 & $(8.4,5.7,3.33,3.34)$ & $(7.8,5.0,3.32,3.29)$ & - & $(8.0,4.8,3.30,3.27)$ \\
APR4-135135 & $(11,7.0,3.40,3.40)$ & - & $(6.6,3.6,3.34,3.34)$ & $(6.5,3.2,3.31,3.31)$ \\
ALF2-120150 & $(4.5,2.5,2.65,2.68)$ & - & $(4.8,2.3,2.75,2.78)$ & $(5.4,2.9,2.70,2.71)$ \\
ALF2-130140 & $(1.7,0.7,2.72,2.72)$ & - & $(1.7,0.9,2.71,2.75)$ & $(1.6,0.8,2.73,2.75)$ \\
ALF2-135135 & $(1.0,0.5,2.77,2.82)$ & - & $(1.5,0.9,2.79,2.82)$ & $(2.8,1.5,2.75,2.76)$ \\
H4-120150 & $(3.5,1.6,2.27,2.27)$ & - & $(3.8,1.8,2.28,2.28)$ & $(3.5,1.8,2.30,2.31)$ \\
H4-135135 & $(0.3,0.1,2.47,2.51)$ & - & $(0.3,0.1,2.48,2.52)(0.5,0.2,2.44,2.48)$ \\
MS1-120150 & $(3.4,1.4,2.08,2.08)$ & - & $(3.1,1.4,2.10,2.09)(3.4,1.5,2.08,2.09)$ \\
MS1-135135 & $(0.6,0.2,2.08,2.07)$ & - & $(0.8,0.3,2.00,1.97)(1.6,0.6,1.98,1.95)$ \\
\hline \hline
\end{tabular}

[49] A. Bauswein and H.-Th. Janka, Phys. Rev. Lett. 108, 011101 (2012).

[50] T.W. Baumgrate, S.L. Shapiro, and M. Shibata, Astrophys. J. Lett. 528, L28 (2000).

[51] G.B. Cook, S.L. Shapiro, and S.A Teukolsky, Astrophys. J. 398, 203 (1992).

[52] E. Berti, V. Cardoso, and A. O. Starinets, Classical Quantum Gravity 26, 163001 (2009).

[53] M. A. Aloy, H.-T. Janka, and E. Muller, Astron. Astrophys. 436, 273 (2005).

[54] http://www.astro.caltech.edu/ptf/

[55] http://pan-starrs.ifa.hawaii.edu/public/

[56] http://www.lsst.org/lsst/

[57] http://www.aoc.nrao.edu/evla/
[58] http://www.atnf.csiro.au/projects/askap/

[59] http://www.ska.ac.za/meerkat/

[60] B. F. Schutz, Nature 323, 310 (1986).

[61] For MS1, for which the maximum mass of spherical neutron stars is quite large (see Table I), the remnant neutron stars are not hypermassive nor supramassive for $m \leq 2.8 M_{\odot}$ (see Refs. [50] and 51] for the definition of the hypermassive and supramassive neutron stars, respectively). We should call the remnant neutron star for this EOS normal massive neutron star (MNS). However, in this paper, we do not distinguish MNS from HMNS for simplicity. 